\title{
Identification of RPS28 as a Promising Therapeutic Target for Osteosarcoma Patients with Poor Prognoses Stratified by a Seven-gene Signature
}

\author{
Yin Sun ${ }^{1,+}$, Juan Zhou ${ }^{2,+}$, Yongjie Wang ${ }^{4,+}$, Lin Zeng ${ }^{3}$, Chao Liang ${ }^{1}$, Jin Zhou ${ }^{1}$, Lan Shao ${ }^{1}$, Zhichang Zhang ${ }^{1}$, Weiping \\ Ji ${ }^{1}$, Yunlong Zhang ${ }^{1}$, Shikui Tu ${ }^{3, *}$, Zhiyan Liu ${ }^{2, *}$ and Yang Dong ${ }^{1, *}$ \\ 1 Department of Orthopaedics, Shanghai Jiao Tong University Affiliated Sixth People's Hospital, Shanghai Jiao \\ Tong University, Shanghai 200233, PR China \\ 2 Department of Pathology, Shanghai Jiao Tong University Affiliated Sixth People's Hospital, Shanghai Jiao \\ Tong University, Shanghai 200233, PR China \\ 3 Department of Computer Science \& Engineering, \& Center for Cognitive Machines \& Computational Health, \\ SEIEE School, Shanghai Jiao Tong University, Shanghai 200240, PR China \\ 4 Department of Orthopaedic Surgery, Shanghai Tenth People's Hospital Affiliated to Tongji University, \\ Shanghai 200072, PR China \\ * Correspondence: tushikui@sjtu.edu.cn (S.T); zhiyanliu@shsmu.edu.cn (Z.L.); dongyang6405@163.com (Y.D.) \\ † These authors contributed equally to this work
}

Simple Summary: Osteosarcoma (OSA) is the most common primary malignant bone tumor. More than $40 \%$ of patients with OSA have poor prognoses. It is essential to discover these high-risk patients and give them more effective therapeutics as soon as they are diagnosed. But no ideal biomarker could be used to separate these high-risk patients from the low-risk patients and guide more precise treatment decisions. To solve this problem, we constructed a seven-gene signature based on the transcriptome data of OSA biopsy specimens, whose prognostic value was validated in the training cohort (88 patients) and two independent cohorts (53 patients and 84 patients, respectively). Additionally, RPS28 was found to promote proliferation and metastasis of OSA cells and simultaneously facilitate immune escape in high-risk patients. Therefore, RPS28 could be a potential therapeutic target worthy of further investigation.

Abstract: Osteosarcoma (OSA) is the most common primary malignant bone tumor. More than $40 \%$ of patients with OSA have poor prognoses. We aimed to discover a biomarker for patient stratification and therapeutic targets for these high-risk patients. Using Single Sample Gene Set Enrichment Analysis (ssGSEA) and univariate Cox analysis, six hallmarks were identified as significant prognostic factors for overall survival (OS). Three were selected to construct a multivariate Cox model. Then, WGCNA, univariate Cox regression, Kaplan-Meier (KM) survival analyses, and multivariate Cox analyses were combined to filter promising candidates and establish a seven-gene signature to predict OS, whose prognostic value was validated internally and externally. Subsequently, Differential Expression Analysis was conducted between high- and low-risk patients, and the Robust Rank Aggregation algorithm was used to determine the robust DEGs. Metascape was used to perform pathway and process enrichment analyses as well as construct protein-protein interaction (PPI) networks. Finally, RPS28 was identified as an independent risk factor by using univariate and multivariate Cox regression, which was preliminarily validated as a promising therapeutic target by using RNA interference. In conclusion, we might contribute to optimizing risk stratification and an excellent therapeutic target for high-risk patients with OSA.

Keywords: osteosarcoma; hallmarks of cancer; predictive model; immune escape; ribosome biogenesis; RPS28 


\section{Introduction}

Osteosarcoma (OSA), the most common primary malignant solid tumor of bone, predominantly occurs in adolescents with a second peak in incidence among adults aged $>65$ years [1]. The standard therapy for OSA, comprising neoadjuvant chemotherapy, surgical removal of the primary lesion along with clinically evident metastatic disease followed by adjuvant chemotherapy, was established in the 1980s and developed gradually, resulting in long-term survival in $>60 \%$ of patients with localized disease [2-4]. Unfortunately, limited progress has been made in improving the survival of patients with OSA [5,6]. As a result, more than $40 \%$ of OSA patients' prognoses are still poor, especially for those who have high-risk factors, including Enneking stage III [7] and Huvos grade I/II [8,9]. However, such qualitative assessments are relatively subjective, time-consuming, and sometimes inaccurate [10]. Additionally, more intensified adjuvant chemotherapy for the patients in high risk separated by Huvos grade was found only increasing toxicity without improving event-free survival [11]. Therefore, it is imperative to discover actionable biomarkers for patient stratification and effective therapeutic targets for high-risk patients with this rare but devastating malignancy.

With the rapid development of high-throughput gene detection technology, such as microarrays and next-generation sequencing, multi-omics data of tumors were available to identify novel diagnostic or prognostic biomarkers as well as therapeutic targets for various types of cancers by applying deep learning to analyze large and disparate datasets from The Cancer Genome Atlas (TCGA) and Gene Expression Omnibus (GEO) [12]. As for OSA, because of its rareness, only a few small datasets are accessed in GEO, and the database named Therapeutically Applicable Research To Generate Effective Treatments (TARGET), which have been used to construct multitudinous signatures to predict the prognosis of patients with OSA, including a four-methylated LncRNA signature [13], hypoxia-associated signature [14], glycolysis-related signature [15], immune-related signature [16-18]. But few of them aimed to identify novel therapeutic targets for further experimental validation. Besides, most of these studies just focused on one kind of molecule or pathway selected according to currently popular areas, with more or less bias, which may compromise the prediction power and reliability.

Weighted Gene Co-expression Network Analysis (WGCNA) is a systems biology method widely used to reveal the relevance of genes and sample traits [19], which was frequently used in OSA datasets to identify the hub genes associated with survival time, metastasis, and alive status [20-22]. However, there are many factors, such as the different follow-up times of each patient, influencing these sample traits. Recently, it was reported to be a reliable method using WGCNA to reveal the hub genes correlated to a linear parameter with prognostic significance, such as the normalized enrichment score (NES) of gene sets $[16,23]$.

In the Molecular Signatures Database (MSigDB), there are fifty hallmark gene sets, which convey specific biological states or processes correlated with cancers, such as P53 PATHWAY, HYPOXIA, and WNT BETA CATENIN SIGNALING [24,25], in which HYPOXIA was identified as a dominant risk factor for overall survival in lung adenocarcinoma patients [23], and seven gene sets were found to be associated with the metastasis status of OSA patients [15]. But few studies reported the relationship between these fifty hallmarks gene sets with the overall survival of OSA patients.

In this study, we attempted to discover the hallmarks with prognostic significance for OSA patients, based on which a robust gene signature for prognosis prediction and patient stratification was constructed. Besides, many efforts were taken to discover and validate the therapeutic targets preliminarily for high-risk OSA patients across three cohorts. Firstly, the NES of the fifty hallmarks in the TARGET-OS cohort were calculated by Single Sample Gene Set Enrichment Analysis (ssGSEA), of which three hallmarks, UV RESPONSE UP, CHOLESTEROL HOMEOSTASIS, and XENOBIOTIC METABOLISM, were found to be independent prognostic factors for overall survival via univariate and multivariate Cox regression, which were used to construct a multivariate Cox model, by which 
a linear parameter of each case, was calculated and named as RiskScoreByGS. Subsequently, WGCNA was used to find the most correlated gene modules to the RiskScoreByGS and the NES of the three hallmarks. Afterward, multiple methods were combined to screen for robust biomarkers and construct a seven-gene signature to separate OSA patients into high- and low-risk groups, which was further validated using microarray datasets GSE21257 and GSE42352. Furthermore, Differential Expression Analysis, Robust Rank Aggregation (RRA) [26], Metascape [27], univariate and multivariate Cox regression analyses were combined to discover novel therapeutic targets for patients in the high-risk group. Finally, multiple platforms and methods were used to preliminarily validate that RPS28 functions as a tumor promoter by promoting proliferation and metastasis of OSA cells and simultaneously facilitating immune escape in the high-risk patients stratified by the signature, making it a potential therapeutic target worthy of further investigation.

\section{Materials and Methods}

\subsection{Datasets Collection and Preparation}

HTseq-Counts, HT-seq FPKM (Fragments Per Kilobase Million), and corresponding clinical data of 88 OSA patients in the TARGET-OS Project were downloaded through the Genomic Data Commons (GDC) Data Transfer Tool (GDC-client_v1.6.1_Windows_x64.zip) from GDC data portal (https://portal.gdc.cancer.gov/). The FPKM value was transformed to TPM (Transcripts per million) value for further analyses. GSE21257 and GSE42352 were downloaded by using $R$ languages (version 4.0.2) via $R$ package "GEOquery" (version 2.58.0). The clinicopathological characteristics of the three cohorts are displayed in Table 1 . In the TARGET-OS cohort, there are 88 cases but only 85 with overall survival time $>0$ and 81 patients with event-free survival time $>0$. In the GSE21257 cohort, there are 53 cases with overall survival time $>0$, of which only 39 patients with event-free survival time $>0$. In the GSE42352 cohort, there are 84 cases without survival time, in which only 53 cases with the information about whether metastasis occurred within five years. Besides, the data of 19 OSA cell lines (OS), 12 mesenchymal stem cells (MSC), and three osteoblasts (OB) in GSE42352 were also used. Additionally, several open access platforms were also used, including R2: Genomics Analysis and Visualization Platform (https://hgserver1.amc.nl/cgi-bin/r2/main.cgi), The Query Gene Function on TIDE (http://tide.dfci.harvard.edu/query) and The Human Protein Atlas (HPA) (https://www.proteinatlas.org/).

Table 1. Clinicopathological characteristics of osteosarcoma patients used in this research

\begin{tabular}{|c|c|c|c|}
\hline & Training Cohort & Validation Cohort & Validation Cohort \\
\hline Characteristics & $\begin{array}{c}\text { TARGET-OS } \\
(\mathrm{N}=88)^{1}\end{array}$ & $\begin{array}{c}\text { GSE21257 } \\
(\mathrm{N}=53)^{2}\end{array}$ & $\begin{array}{c}\text { GSE42352 } \\
(\mathrm{N}=84)^{3}\end{array}$ \\
\hline $\begin{array}{l}\text { Age (median }\left[\mathrm{IQR}^{7}\right] \text { ) } \\
\text { (years) }\end{array}$ & $15.00[13.00,18.00]$ & $17.00[14.00,19.00]$ & $16.00[14.00,19.50]$ \\
\hline \multicolumn{4}{|l|}{ Gender $(\%)$} \\
\hline Female & $37(43.0)$ & $19(35.8)$ & $29(34.9)$ \\
\hline Male & $49(57.0)$ & $34(64.2)$ & $54(65.1)$ \\
\hline \multicolumn{4}{|l|}{ Response (\%) } \\
\hline good & $18(41.9)$ & $18(38.3)$ & $33(46.5)$ \\
\hline poor & $25(58.1)$ & $29(61.7)$ & $38(53.5)$ \\
\hline \multicolumn{4}{|l|}{ Dead (\%) } \\
\hline no & $57(66.3)$ & $30(56.6)$ & NA \\
\hline yes & $29(33.7)$ & $23(43.4)$ & NA \\
\hline $\mathrm{OS}^{4}$ (median [IQR]) & $47.00[21.00,69.00]$ & $45.00[27.00,94.00]$ & NA \\
\hline
\end{tabular}




\begin{tabular}{lccc}
\hline $\begin{array}{l}\text { (months) } \\
\text { Relapse }^{5} \text { (\%) }\end{array}$ & & & \\
$\quad$ no & $44(53.7)$ & $19(35.8)$ & $19(35.8)$ \\
$\quad$ yes & $38(46.3)$ & $34(64.2)$ & $34(64.2)$ \\
$\begin{array}{l}\text { EFS } \\
\text { (months) }\end{array}$ & $22.50[13.00,63.00]$ & $21.00[0.00,77.00]$ & NA \\
$\begin{array}{l}\text { Enneking Stage (\%) } \\
\quad \text { III }\end{array}$ & $22(25.0)$ & $14(26.4)$ & \\
$\quad$ non-III & $66(75.0)$ & $39(73.6)$ & NA \\
\hline
\end{tabular}

${ }^{1}$ Only 85 cases with overall survival time $>0$; Only 81 cases with event-free survival time $>0$.

${ }^{2}$ Only 39 cases with event-free survival time $>0$.

${ }^{3}$ Only 53 cases with the information about whether metastasis occurred within five years

4. Overall survival

5. Local or distant recurrence

6. Event-free survival, Event: local or distant relapse

7 Interquartile Range

\subsection{Single Sample Gene Set Enrichment Analysis (ssGSEA) and Selection of Significant} Hallmarks

The fifty hallmark gene sets were downloaded from the Molecular Signatures Database (MSigDB) [25]. The normalized enrichment score (NES) of each hallmark of the 88 cases in the TARGET-OS cohort were quantified by the ssGSEA algorithm via R package "gsva" (version 1.38.2) based on transcriptome profiling data presented as log2 (TPM+1) . The significance of different hallmarks in the TARGET-OS cohort was evaluated via univariate Cox regression for overall survival using the R package "survival" (version 3.1-12). With a threshold of $\mathrm{p}$-value $<0.05$, the significant hallmarks were selected for the following analysis.

\subsection{Construction and Validation of Multivariate Cox Model Based on Hallmarks}

The significant hallmarks mentioned above were screened according to the Akaike information criterion (AIC) [28] by using the R package "my. stepwise" (version 0.1.0). Finally, using the R package "survival", selected hallmarks were used to construct a multivariate Cox proportional-hazard model based on 85 cases with overall survival time $>0$. Then, the RiskScoreByGS of all patients was calculated using the function: predict (model, data). The patients with appropriate prognostic information were divided into two groups using the median of RiskScoreByGS as the cut-off value. The Kaplan-Meier (KM) survival analysis was used to assess the clinical predictive capacity of the RiskScoreByGS via "survminer" packages (version 0.4.9).

2.4. Weighted Gene Co-expression Network Analysis (WGCNA) and Selection of the Highest correlated modules

In the TARGET-OS dataset, 88 cases with RiskScoreByGS and the NESs of the three hallmarks were used for performing WGCNA to identify the modules most correlated with the RiskScoreByGS and each hallmark. The TPM matrix of 19690 mRNAs in 88 cases was constructed, in which 6726 mRNAs, whose TPM values were 0 in more than 44 cases, were removed. Finally, $12964 \mathrm{mRNAs}$ with median absolute deviation (MAD) > 0.01 were selected as input data for subsequent WGCNA using the R package "wgcna" (version 1.703). No outlier samples were detected and excluded with sample hierarchically clustering 
method before six was selected as an appropriate soft threshold power for achieving standard scale-free networks. Furthermore, via the construction of adjacency and topological overlap matrix (TOM) and the calculation of corresponding dissimilarity (1-TOM), gene dendrogram and module identification were achieved with dynamic tree cut, and the minimum module size was 30 . Additionally, clustering of module eigengenes was conducted to merge highly similar modules with dissimilarity $<0.25$. The correlation between module eigengene and sample traits, including RiskScoreByGS, as well as the NESs of UV RESPONSE UP, CHOLESTEROL HOMEOSTASIS, and XENOBIOTIC METABOLISM, was calculated. Finally, two modules were selected for the following analyses.

\subsection{Establishment and Validation of Multivariate Cox Model Based on Gene Signature Related to the RiskScoreByGS}

The genes of two selected modules were conducted a large-scale survival analysis using the R package "survival". With a threshold of the p-value of univariate Cox regression $<0.01$ as well as the $p$-value of log-rank test $<0.01,43$ genes were selected and used as candidates to be further filtered according to Akaike information criterion (AIC) [28]. Finally, selected genes were used to construct a multivariate Cox proportional-hazard model to predict overall survival (OS) using the R package "survival". The Risk Score was calculated by using a function: predict (model, data). OSA patients were divided into two groups by using the median Risk Score as the cut-off value. The Kaplan-Meier survival analysis was used to assess the clinical predictive capacity of the Risk Score in the whole cohort and subgroups via "survminer" packages (version 0.4.9). Time-dependent receiver operating characteristic curves (tROC) was performed to measure the predictive power, and the areas under the curve at different time points [AUC(t)] of our signature and other clinicopathological prognostic factors were compared. Furthermore, the value of patients stratification of this signature was validated in GSE21257 and GSE42352, in which, R package "ggstatsplot" (version 0.8.0) was used to conduct Chi-square test and visualization. Finally, based on this multivariate Cox model, a nomogram and a calibration curve were plotted using the R package "regplot" (version 1.1) and "rms" (version 6.2-0), respectively.

\subsection{Differential Expression Analysis and Robust Rank Aggregation Analysis}

The patients in TARGET-OS, GSE21257, and GSE42352 were separated into low- and high-risk groups according to the respective median of Risk Score predicted based on the multivariate Cox model mentioned above. Differential Expression Analysis was conducted between high-risk cases and low-risk cases by using the R package "limma" (version 3.46.0) and "edgeR" (version 3.32.1). To integrate the three datasets, we used Robust Rank Aggregation (RRA) method to determine the robust differential expressed genes (DEGs) by using the R package "RobustRankAggreg" (version 1.1), which was reported as a standard method to minimize the bias and errors among several datasets [29]. Genes with the mean of $\mid \log 2$ (fold change) $\mid>0.69$ and p-value $<0.05$ were considered as the significant robust DEGs. Finally, the Metascape database (https://metascape.org/) [27] was used for functional and pathway enrichment analyses. A min overlap $\geqslant 3 \& \mathrm{p}$-value $\leqslant$ 0.01 was considered statistically significant. Protein-protein interaction (PPI) enrichment analyses were carried out and visualized, and Molecular Complex Detection (MCODE) algorithm was applied to identify densely connected network components.

\subsection{Assessment of Immune Infiltration}

R package "estimate" (version 1.0.13) [30] was used to calculate ImmuneScore, StromalScore, EstimateScore, and TumorPurityScore, whose correlation with Risk Score as well as RPS28 was calculated and visualized using R package "ggpubr" (version 0.4.0). The relative enrichment scores of 24 immune cell types in the tumor microenvironment 
were estimated using the Immune Cell Abundance Identifier (ImmuneCellAI) [31], whose correlation with down-regulated genes was further analyzed and visualized by using the R package "pheatmap" (version 1.0.12) with a little modified.

\subsection{Immunohistochemistry}

OSA tissues along with para-tumor soft tissues were obtained from five OSA patients who have been conducted limb-sparing or amputation surgery from January to June in 2021, whose corresponding normal bone tissues were harvested from the broken-end of tumor segments. This study followed standard guidelines and was approved by the Ethics Committee of Shanghai Jiao Tong University Affiliated Sixth People's Hospital, Shanghai, PR China. Written informed consent was obtained from all participants, whose demographic information and clinicopathological characteristics were displayed in Table 2. OSA tissues were fixed in $4 \%$ paraformaldehyde. Bone tissues were decalcified in $10 \%$ ethylenediaminetetraacetic acid decalcification solution. All tissues were dehydrated, embedded in paraffin. Subsequently, four $\mu$ m-thick tissue sections were stained with primary antibodies against RPS28 (Sigma-Aldrich Cat\# HPA047132, dilution 1:100, Saint Louis, State of Missouri, USA) and a horseradish peroxidase-conjugated IgG (Servicebio, Wuhan, China). The staining results were shown by diaminobenzidine (DAB), scanned using the NDP NanoZoomer S210 (Hamamatsu, ShizuokaPref, Japan), and analyzed with the NDP view 2.0 software.

Table 2. Demographic information and clinicopathological characteristics of the patients in our cohort.

\begin{tabular}{cccccccccc}
\hline Ethnicity & Gender & Age & $\begin{array}{c}\text { Tumor } \\
\text { side }\end{array}$ & $\begin{array}{c}\text { Tumor } \\
\text { site }\end{array}$ & $\begin{array}{c}\text { Tumor } \\
\text { region }\end{array}$ & $\begin{array}{c}\text { Eenneking } \\
\text { stage }\end{array}$ & Surgery & $\begin{array}{c}\text { Tumor } \\
\text { necrosis } \\
\text { rate }\end{array}$ & $\begin{array}{c}\text { Pathological } \\
\text { diagnosis }\end{array}$ \\
\hline Han & Male & 38 & Right & Femur & Distal & III & Limb-sparing & $85 \%$ & Classical OSA \\
Han & Male & 20 & Right & Femur & Distal & III & Amputation & $5 \%$ & Classical OSA \\
Han & Male & 42 & Left & Femur & Proximal & IIB & Limb-sparing & $30 \%$ & Classical OSA \\
Han & Female & 10 & Right & Tibia & Proximal & IIB & Limb-sparing & $15 \%$ & Classical OSA \\
Han & Female & 9 & Left & Tibia & Proximal & IIB & Limb-sparing & $66 \%$ & Classical OSA \\
\hline
\end{tabular}

\subsection{Cell Line and Cell Culture}

As described before [32], all of the human OSA cell lines were purchased from the Chinese Academy of Sciences (Shanghai, China) and cultured in Dulbecco's modified Eagle's medium (DMEM) (Hyclone, Tauranga, New Zealand) with $10 \%$ fetal bovine serum (FBS) (Gibco, NY, USA), as well as penicillin $(100 \mathrm{U} / \mathrm{ml})$ and streptomycin $(100 \mu \mathrm{g} / \mathrm{ml})$ (Invitrogen, $\mathrm{CA}, \mathrm{USA}$ ) at $37^{\circ} \mathrm{C}$ in a humidified $5 \% \mathrm{CO}_{2}$ atmosphere.

\subsection{RNA extraction and quantitative real-time-PCR}

Total RNA was isolated using TRIzol Reagent (Life Technologies, CA, USA), then used to synthesize complementary DNA using the iScript cDNA Synthesis Kit (Bio-Rad, CA, USA). Subsequently, quantitative real-time PCR was conducted using SYBR premix Ex Taq (Takara, Liaoning, China) and ABI 7500 Sequence Detection System (Thermo Fisher Scientific, MA, USA). All procedures were performed according to the manufacture's protocols. The sequences of primers used are listed as follows: GAPDH, forward, 5'-GGAGCGAGATCCCTCCAAAAT-3' and reverse, 5'-GGCTGTTGTCATACTTCTCATGG-3'; RPS28, forward, 5'-GACACGAGCCGATCCATCATC-3' and reverse, 5'-TGACTCCAAAAGGGTGAGCAC-3'. 


\subsection{RNA Interference}

RPS28 siRNA duplexes and corresponding si-control were purchased from RiboBio (Guangzhou, China). The sequences targeting RSP28 were as follows: si-RPS28-1, 5'CCATCATCCGCAATGTAAA-3'; si-RPS28-2, 5'-GCTCACCCTTTTGGAGTCA-3'; siRPS28-3, 5'-TGCGCGTGGAATTCATGGA-3'. According to the manufacturer's protocol, transient transfections were performed with Lipofectamine 3000 (Invitrogen, CA, USA).

\subsection{Western Blotting analysis}

Protein was extracted from RPS28-knockdown and control MG-63 cells using RIPA lysis buffer (CST, MA, USA), whose concentration was assessed using the Bicinchonimic acid Protein Assay Kit (Pierce, IL, USA). Cell homogenates containing equal amounts of protein were separated on 10\% SDS-PAGE and then transferred on $0.22-\mu \mathrm{m}$ polyvinylidene difluoride membranes, which were blocked with $5 \%$ skimmed milk for one hour at room temperature. After being incubated with primary antibody (1:1000) overnight at four ${ }^{\circ} \mathrm{C}$, the membranes were washed twice with TBST and incubated with horseradish peroxidase- (HRP) linked secondary antibodies (1:5000) at room temperature for one hour. ECL Kit (Servicebio, Wuhan, China) was used to visualize the blots. The primary antibodies used were GAPDH Rabbit mAb (A19056) (Abclonal, Wuhan, China) and RPS28 Rabbit pAb (A17937) (Abclonal, Wuhan, China). The secondary antibody used was HRP Goat Anti-Rabbit IgG (H+L) (AS014) (Abclonal, Wuhan, China).

\subsection{Cell Counting Kit-8 Assay and Colony Formation Assay}

Cell counting kit-8 (CCK-8) and colony formation assays were conducted to assess the proliferation of OSA cells. For the CCK-8 assay, RPS28-knockdown and control MG63 cells were seeded in 96-well plates in triplicate at a density of $3 \times 10^{3}$ cells/well. The cell viability was evaluated quantitatively at the indicated timepoints by adding $100 \mu \mathrm{L}$ fresh culture medium with 10\% CCK-8 reagent (Dojindo Laboratories Co., Ltd., Kumamoto, Japan) to each well after removing the old medium, followed by incubation at $37^{\circ} \mathrm{C}$ for 1 hour and detecting the optical density (O.D.) at $450 \mathrm{~nm}$ by using a microplate reader (BIOTEK, Vermont, USA). Finally, each O.D. value was normalized to the control group's average to obtain the ratio of viable cells, as published previously [32].

For the colony formation assay, RPS28-knockdown and control MG-63 cells were seeded in $6 \mathrm{~cm}$ dishes in triplicate at a density of $1 \times 10^{3}$ cells/dish, then cultured with the medium replaced every three days. After one week, colonies were fixed with $4 \%$ paraformaldehyde, stained with $1 \%$ crystal violet, washed with phosphate-buffered saline (PBS) solutions, and photographed. The number of clones with more than 50 cells was counted.

\subsection{Wound Healing, Transwell Migration, and Matrigel Invasion Assays}

For the wound healing assay, RPS28-knockdown and control MG-63 cells were seeded in 6-well plates in triplicate. Cells were scratched with a $200 \mu \mathrm{L}$ sterile pipette tip to make a gap when the cell density reached approximately $90 \%$, which were further cultured in serum-free medium for 24 hours. The gaps were observed and photographed at 0 and 24 hours by CKX41 inverted microscopy (Olympus, Tokyo, Japan) and analyzed with Fiji Image J Software.

For transwell migration assay, cells were pretreated with $10 \mathrm{ug} / \mathrm{ml}$ mitomycin C (Selleck Chemicals, TX, USA) for two hours. Then, $5 \times 10^{4}$ cells with $100 \mu \mathrm{L}$ serum-free DMEM were seeded into the upper transwell ${ }^{\circledR}$ chamber (Corning \#3422) (Corning, NY, USA), whose lower chamber was added $600 \mu \mathrm{L} \mathrm{DMEM} \mathrm{with} 10 \%$ FBS. After incubation for $48 \mathrm{~h}$, cells in the bottom chamber were fixed with $4 \%$ paraformaldehyde, stained with 
$1 \%$ crystal violet, washed with PBS solutions, and photographed. Three random fields of vision were captured and counted the fixed cells.

Matrigel invasion assay was carried out with Corning ${ }^{\circledR}$ BioCoat ${ }^{\mathrm{TM}}$ Matrigel Invasion Chambers (\#354480)( Corning, NY, USA). Briefly, $5 \times 10^{4}$ cells with $100 \mu \mathrm{L}$ serum-free DMEM were seeded into the upper chamber, whose lower chamber was added $600 \mu \mathrm{L}$ DMEM with $10 \%$ FBS. After incubation for $72 \mathrm{~h}$, cells in the bottom chamber were stained, imaged, and counted as the same methods in the migration assay.

\subsection{Statistical and Other Computational Analyses}

Unless stated otherwise, statistical analyses were conducted based on the $\mathrm{R}$ language (version 4.0.2) (https://cran.r-project.org/) and Graphpad Prism Version 9.0 software. All experiments were performed at least three times independently, and results are expressed as means \pm standard deviation (S.D.). The student's t-test was used to analyze differences between the two groups. The differences were considered significant if $p$-value $<0.05$.

\section{Results}

\subsection{Schematic Diagram of the Research Design}

Firstly, six hallmark gene sets are identified as significant risk or protective factors for overall survival in OSA patients. Three were selected to construct a multivariate Cox model based on gene sets (Figure 1a). Then, WGCNA, univariate Cox regression analysis, Kaplan-Meier (KM) survival analysis, and multivariate Cox regression were combined to filter promising candidates and establish a gene signature to predict overall survival (Figure $1 \mathrm{~b}$ ). Subsequently, the prognostic value of the gene signature was assessed and validated in the training and two independent validation cohorts (Figure 1c). Differential Expression Analysis was conducted between high- and low-risk patients, separated according to the median Risk Score in three independent cohorts, respectively. The Robust Rank Aggregation method was used to determine the robust DEGs. The upregulated and downregulated DEGs were respectively imported into Metascape to perform pathway and process enrichment analyses as well as construct protein-protein interaction (PPI) networks, in which the Molecular Complex Detection (MCODE) algorithm has been applied to identify densely connected network components, which may be the critical therapeutic targets, in which RPS28 was identified as an independent prognostic factor (Figure 1d). Finally, RPS28 was preliminarily validated as a promising therapeutic target using multiple methods (Figure 1e). 
a. Construct multivariate Cox model based on hallmark gene sets

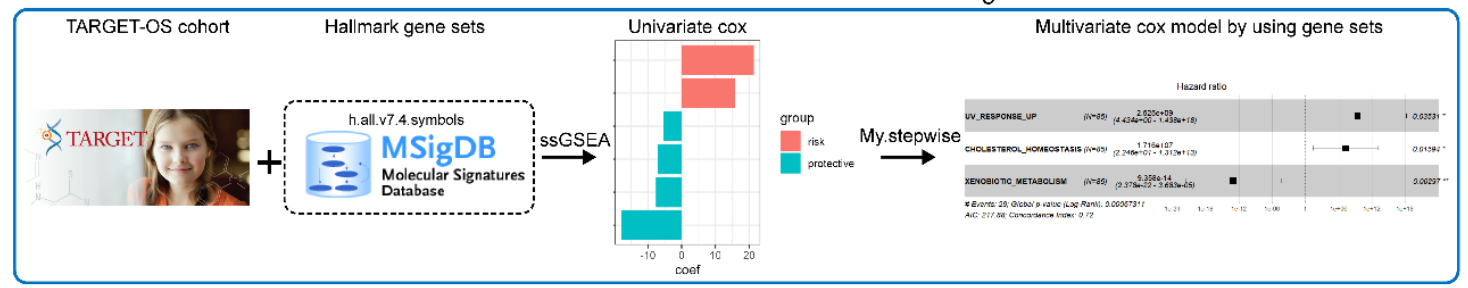

b. Establish multivariate Cox model based on gene signature related to the significant hallmark gene sets

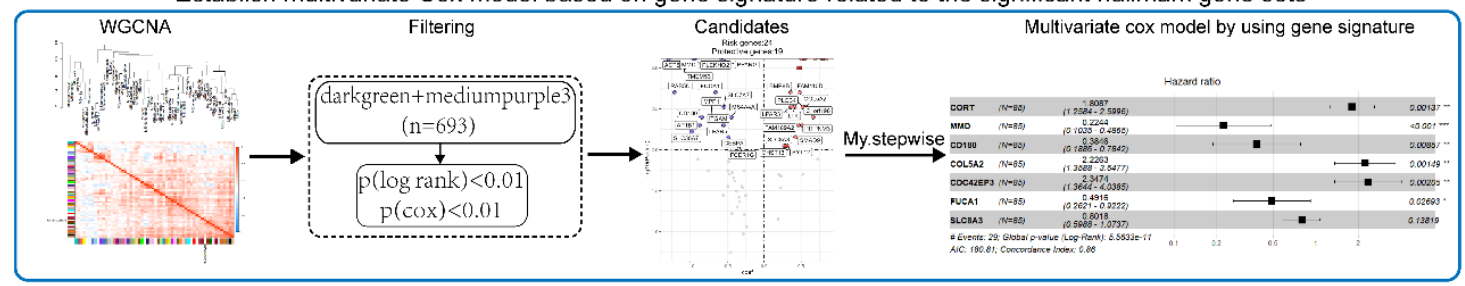

C. Assess and validate the prognostic value of the mutivariate Cox model based on gene signature

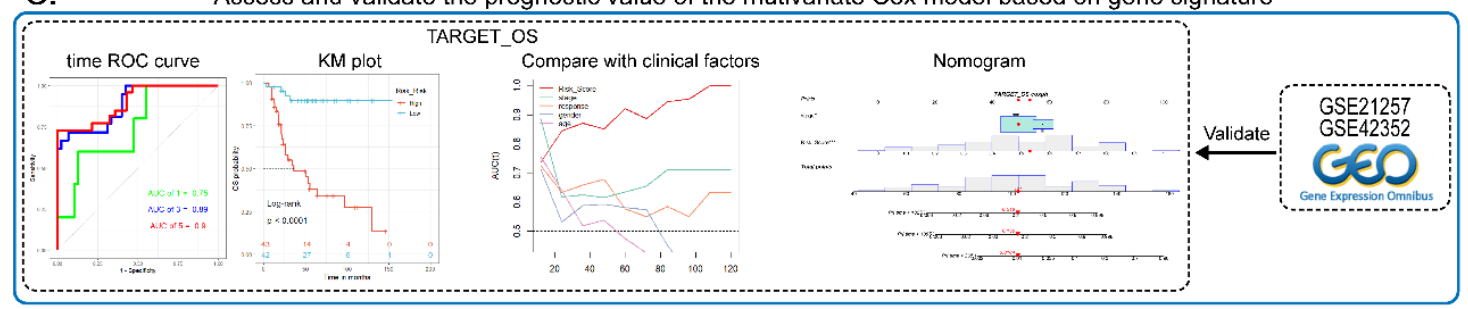

d.

Identify therapeutic targets for patients in the high-risk group across the three cohorts

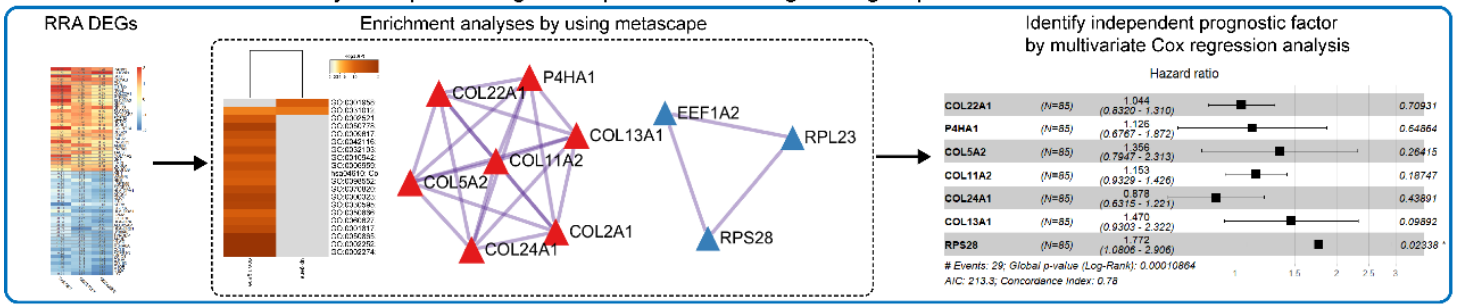

e.

Preliminarily validate RPS28 as promising theraputic target using multiple methods

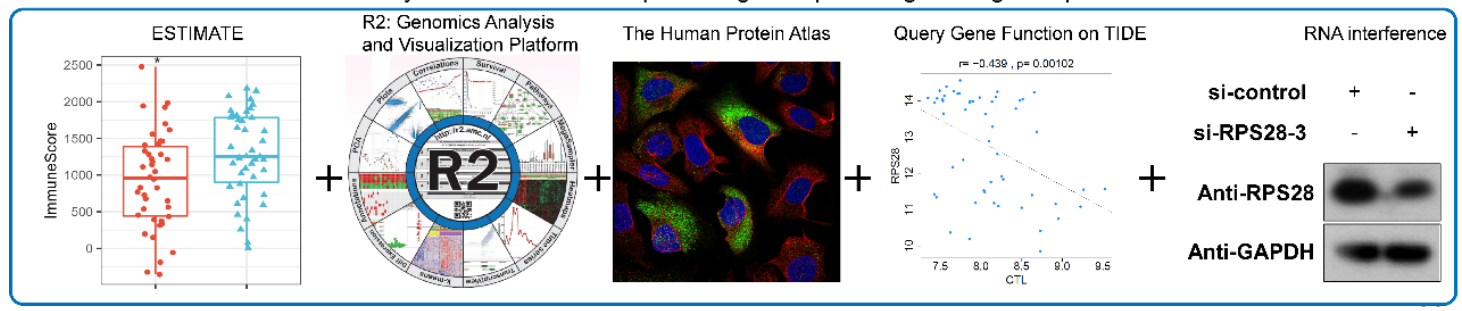

Figure 1. Schematic diagram of the research design. (a) Three hallmarks were selected to construct a multivariate Cox model for overall survival in OSA patients; (b) Combined methods were conducted to establish a gene signature for prognosis; (c) Assess and validate the predictive value of the gene signature; (d) Identify therapeutic targets for patients in the high-risk group separated by the gene signature; (e) Preliminarily validate RPS28 as promising therapeutic target using multiple methods.

\subsection{Construction of Multivariate Cox Proportional-Hazard Model Based on Hallmark Gene Sets}

Based on the NES of cancer hallmarks and overall survival information in the TARGET-OS cohort, the Cox coefficient of each hallmark was calculated. With the threshold of the p-value of univariate Cox regression $<0.05$, six significant hallmarks were discovered and displayed (Figure 2a), in which three were selected further to construct a multivariate Cox proportional-hazard model, including UV RESPONSE UP, CHOLESTEROL 
HOMEOSTASIS, and XENOBIOTIC METABOLISM (Figure 2b). The RiskScoresByGS of 85 samples in this cohort were calculated and ranked, of which the median was used as the cut-off value to divide 85 patients into low-risk and high-risk groups (Figure 2c). After that, the overall survival status of the two groups of patients was displayed and compared (Figure 2d, Figure2f). A heatmap was drawn to illustrate the profiles of the three hallmarks in all samples (Figure 2e). Kaplan-Meier (KM) curves demonstrated that low-risk patients had better survival than high-risk ones $(\mathrm{p}=0.0017)$, showing that RiskScoreByGS has a practical value for predicting overall survival (Figure $2 \mathrm{f}$ ). The same analyses were conducted in 81 patients whose event-free survival data could be obtained, further validating the RiskScoreByGS had a remarkable prognostic value (Figure 2g-2j).

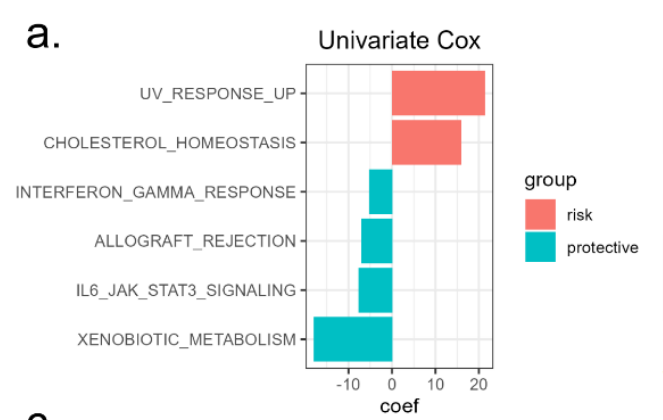

C.

d.
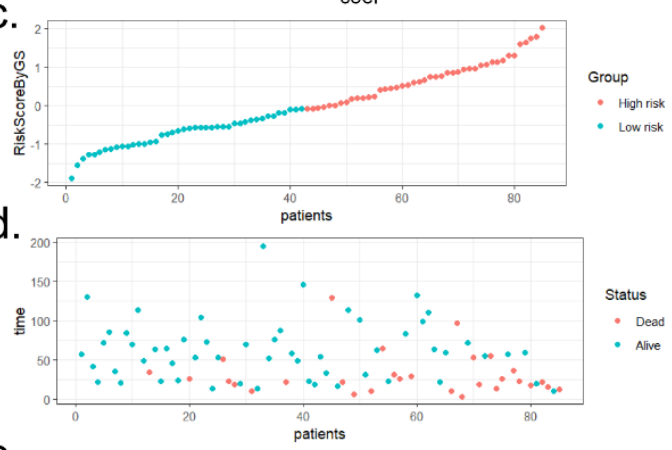

e.

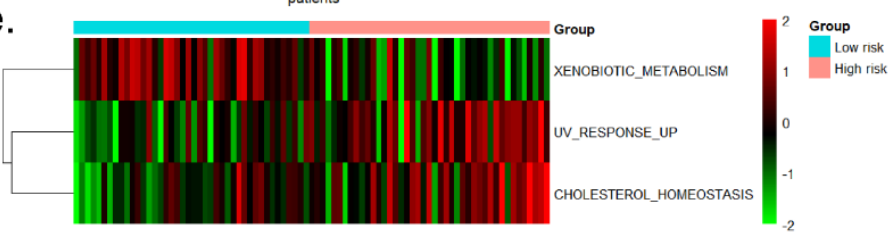

f.

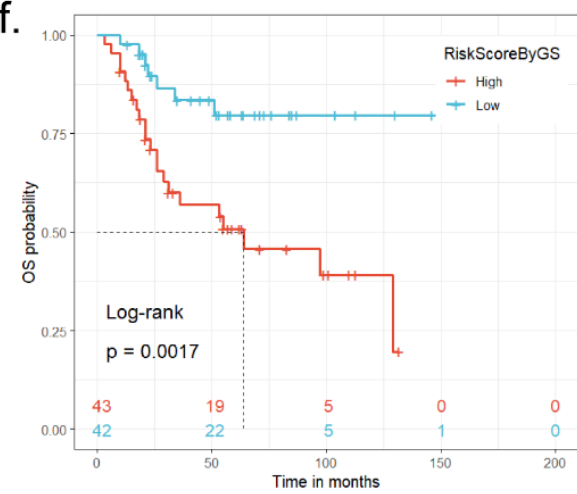

b.

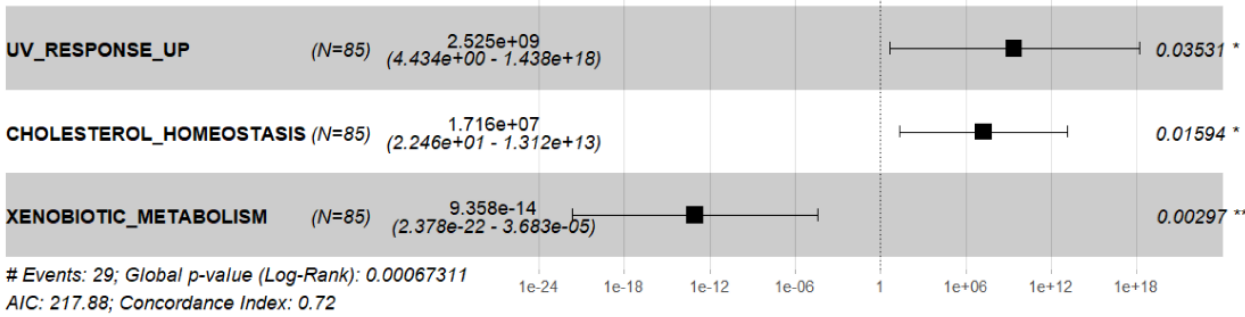

g.

h.

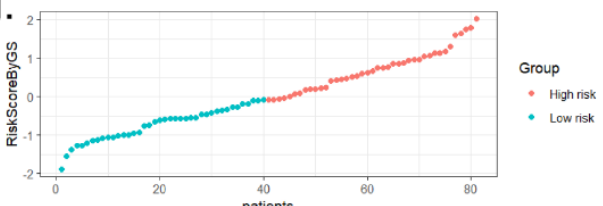

h.

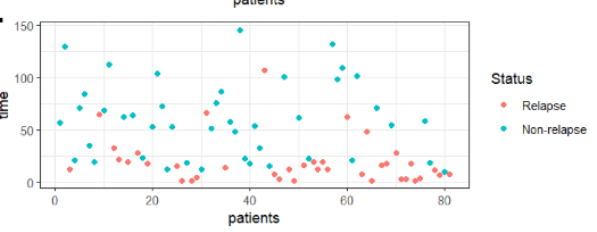

i.

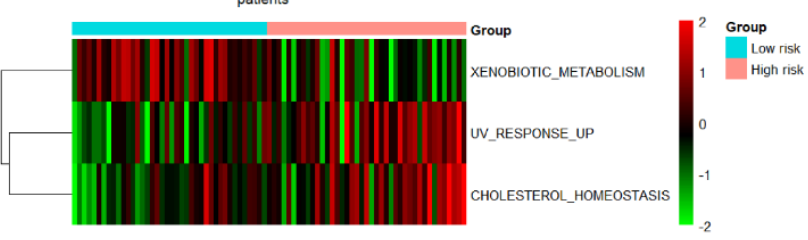

j.

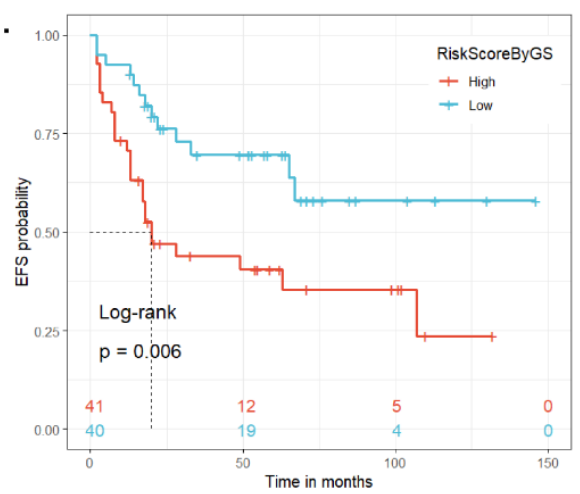

Figure 2. Construction of multivariate Cox model based on hallmark gene sets. (a) Six hallmarks of cancer were found correlated with the overall survival of OSA patients using univariate Cox regression analysis; (b) Three hallmarks were selected to construct a multivariate Cox model for overall survival; (c, g) Distribution of RiskScoreByGS in high- and lowrisk group. Overall survival status $(\mathrm{d})$ and event-free survival status $(\mathrm{h})$ between the high- and low-risk group; (e, i). Heatmap of the profile of three hallmarks. Kaplan-Meier survival curve of overall survival (f) and event-free survival (j) between the low- and high-risk group. 
3.3. Establishment of Multivariate Cox Proportional-Hazard Model Based on Gene Signature Related to the Significant Hallmark Gene Sets

WGCNA was conducted using transcriptome profiling data of 88 samples in the TARGET-OS cohort to find the modules related to the RiskScoreByGS and the NESs of the three significant hallmark gene sets (Figure S1a). $\beta=6$ and $\mathrm{R} 2=0.85$ were recognized as the soft-threshold for scale-free network (Figure S1b). The minimum module size was set at 30, and modules with a correlation coefficient greater than 0.75 were merged. Subsequently, 58 modules were set up (Figure S1c). Module-trait relationships' heatmaps (Figure 3a) demonstrated that the module most negatively correlated with RiskScoreByGStrait (correlation coefficient $=-0.39, p=0.0002$ ), mediumpurple3, was most positively correlated with XENOBIOTIC METABOLISM-trait (correlation coefficient $=-0.41, \mathrm{p}=7 \mathrm{e}-05$ ), and the module most positively correlated with RiskScoreByGS-trait (correlation coefficient $=0.51, p=7 e-05)$, dark green, was most positively correlated with UV RESPONSE UP-trait (correlation coefficient $=0.32, p=0.002$ ) as well as CHOLESTEROL HOMEOSTASIS-trait (correlation coefficient $=-0.34, p=0.001$ ). Gene significances of RiskScoreByGStrait versus two module memberships were displayed in Figure 3b-3c. The genes in two modules were combined, and conducted a large-scale survival analysis in 85 patients whose overall survival information could be obtained. With a threshold of the p-value of univariate Cox regression $<0.01$ and the $p$-value of log-rank test $<0.01,43$ genes were selected, displayed in Figure 3d, and used as candidates to be further filtered. Finally, seven genes (Table 3 ) were selected to construct a multivariate Cox proportional-hazard model for predicting overall survival, with a global p-value and concordance index of $5.5633 \mathrm{e}-11$ and 0.86 , respectively (Figure $3 \mathrm{e}$ ).

Table 3. Univariate Cox and log-rank test.

\begin{tabular}{llcccc}
\hline Gene & \multicolumn{1}{c}{ Description } & H.R. & $\mathbf{9 5 \%} \mathbf{C l}$ & $\boldsymbol{P}$. value & $\boldsymbol{P}$. value of log-rank \\
\hline MMD & monocyte to macrophage differen- & 0.385 & $(0.23-0.64)$ & 0 & 0.002276441 \\
& tiation associated & 0.429 & $(0.24-0.76)$ & 0.004 & 0.003298091 \\
CD180 & CD180 molecule & 0.519 & $(0.34-0.78)$ & 0.002 & 0.006954024 \\
FUCA1 & alpha-L-fucosidase 1 & 1.313 & $(1.07-1.61)$ & 0.009 & 0.007659437 \\
SLC8A3 & solute carrier family 8 member A3 & 1.653 & $(1.30-2.11)$ & 0 & 0.001457926 \\
CORT & cortistatin & 1.761 & $(1.21-2.55)$ & 0.003 & 0.003363175 \\
COL5A2 & collagen type V alpha 2 chain & 2.211 & $(1.42-3.45)$ & 0 & 0.003927561 \\
CDC42EP3 & CDC42 effector protein 3 & & & & \\
\hline
\end{tabular}



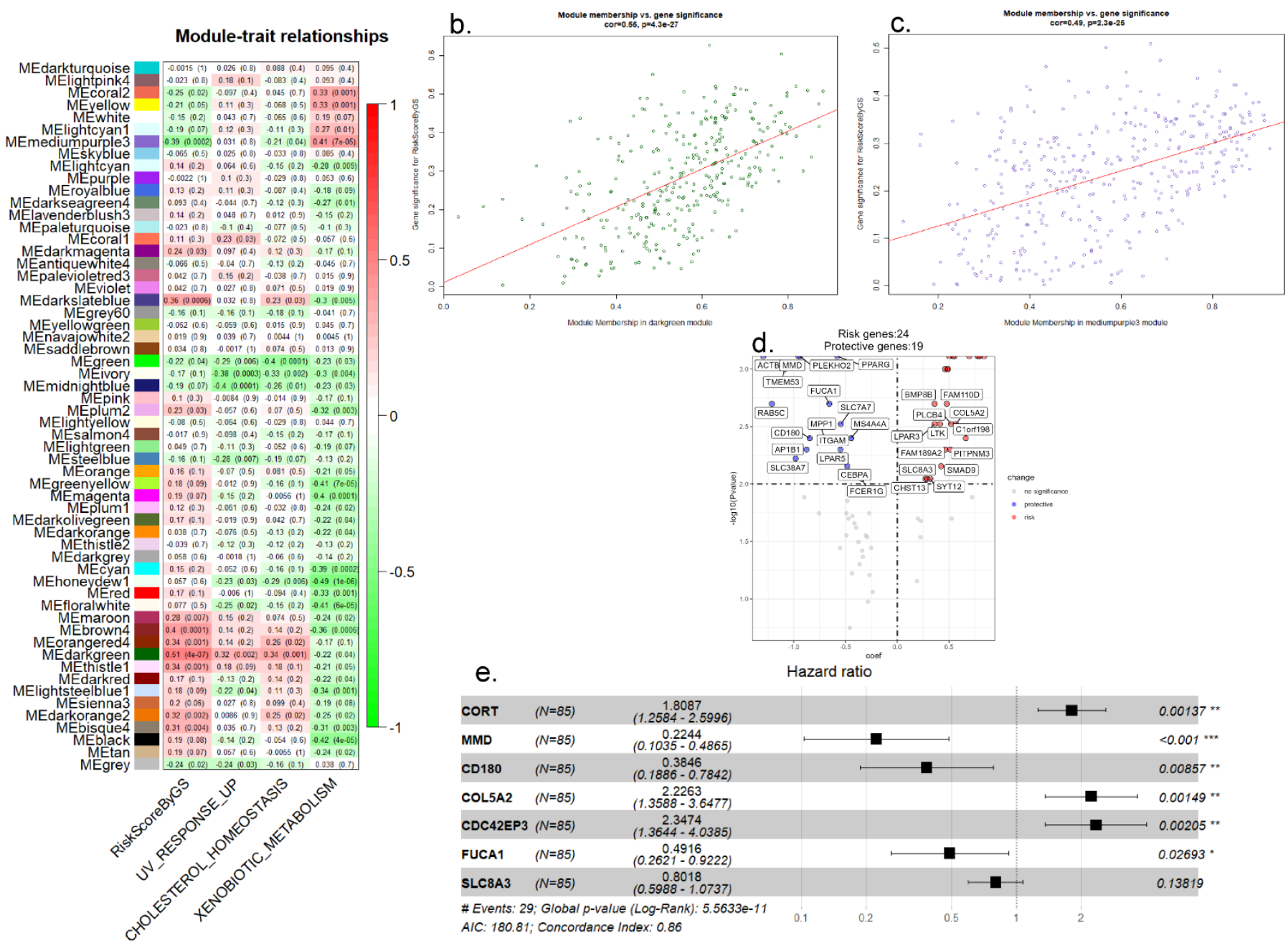

Figure 3. Establishment of multivariate Cox model based on gene signature related to RiskScoreByGS. (a) Heatmaps of the module-trait relationship between gene modules and traits of RiskScoreByGS and the NESs of three hallmarks; Scatter plot of genes in dark green module (b) and mediumpurple3 module (c); (d) Volcano plot showing the significant genes in dark green and mediumpurple3 modules; (e) Forest plot displaying Hazard ratio of seven multivariate Cox candidates genes for overall survival.

\subsection{Assessment and Validation of the Prognostic Value of Multivariate Cox Proportional-Hazard} Model Based on Gene Signature

According to the multivariate Cox proportional-hazard model based on the sevengene signature, Risk Scores of 85 samples in the TARGET-OS cohort were calculated and ranked. The median was used as the cut-off value to separate 85 patients into low-risk and high-risk groups (Figure 4a). After that, the overall survival status of the two group patients was displayed and compared (Figure $4 \mathrm{~b}$, Figure $4 \mathrm{~d}$ ). A heatmap was drawn to illustrate the expression profiles of the seven genes in the corresponding samples (Figure 4c). Kaplan-Meier (KM) curves demonstrated low-risk patients had remarkably better survival than high-risk ones $(p<0.0001)$ (Figure $4 d)$. Time-dependent ROC curves indicated that this multivariate Cox model had good robustness with a 1-year AUC of 0.75, 3-years AUC of 0.89 , and 5-years AUC of 0.9 (Figure 4e). When compared with clinicopathological characteristics, including gender, age, stage, and response to neoadjuvant chemotherapy, the prognostic value of this gene signature exhibited the most potent and stable ability for predicting overall survival most of the time (Figure 4f).

To further evaluate the independence and applicability of this seven-gene signature, patients were separated into different subgroups according to clinicopathological characteristics and performed KM survival analysis. The KM curves showed that regardless of 
gender, age, stage, and response to neoadjuvant chemotherapy, significant differences between the overall survival of low-risk and high-risk patients were observed (Figure 5a$5 \mathrm{~h})$. The same analyses were conducted in 81 patients whose event-free survival data could be obtained, further validating this gene signature had a remarkable prognostic value (Figure $4 \mathrm{~g}-4 \mathrm{i}$ and Figure $5 \mathrm{i}-5 \mathrm{p}$ ).

GEO datasets, GSE21257 and GSE42352, were used as independent validation cohorts to ensure the reliability of this seven-gene signature. GSE21257 comprised 53 cases, in which 27 were separated into high-risk groups and 26 were in the low-risk group. KM curve showed that the low-risk group had a better overall survival $(p=0.028)$ (Figure S2a). Time-dependent ROC curves demonstrated that this signature also had good robustness in an independent cohort with a 1-year AUC of 0.82, 3-years AUC of 0.72, and 5-years AUC of 0.77 (Figure S2b). In GSE21257, only 39 cases whose event-free survival time was more than 0 months were used to validate the prognostic value for predicting event-free survival (Figure S2c-S2d). In GSE42352, the patients who relapsed within five years had a higher risk score than those non-relapsed $(p=0.0019)$ (Figure S2e). Additionally, 85\% of patients relapsed within five years in the high-risk group, significantly higher than that in the low-risk group (Chi-square test $\mathrm{p}=0.002$ ) (Figure S2f). These results demonstrated that this seven-gene signature was an excellent prognostic instrument, including overall and event-free survival in validation cohorts.

Enneking staging system is a known prognostic factor for OSA patients, which was combined with Risk Score to conduct multivariate Cox regression to verify the independence of Risk Score in estimating prognosis (Figure 6a and 6b). Nomogram was a powerful method to integrate different risk factors to predict the prognosis of patients with tumors. Therefore, The multivariate Cox model using seven-gene signature was visualized by using nomogram (Figure 6c), whose calibration curve was plotted in Figure 6d, illustrating that the nomogram predicted 5-year overall survival better than 1- and 3- year overall survival for their relative more minor divergence between actual and predicted survival status (Figure 6d).

a.

b.

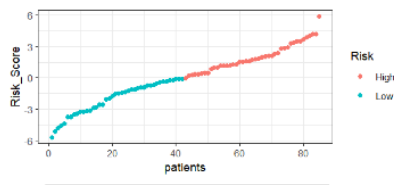

b.

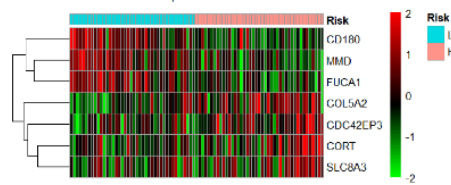

g.

h.

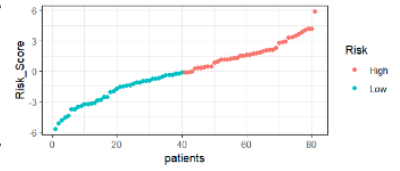

d.

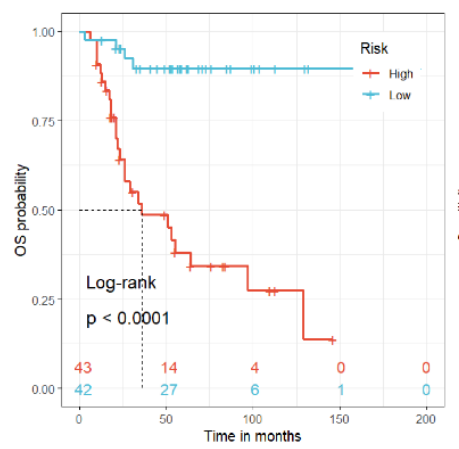

j.

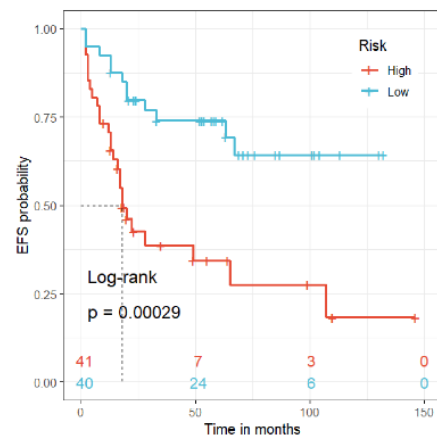

e.

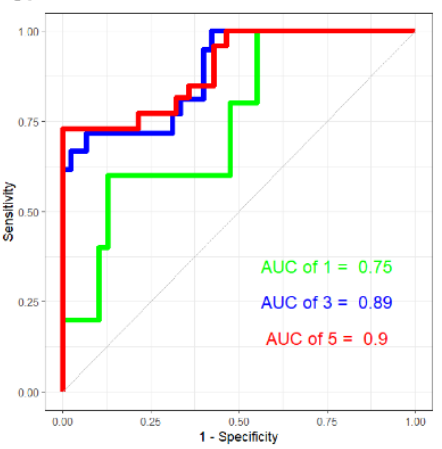

k.

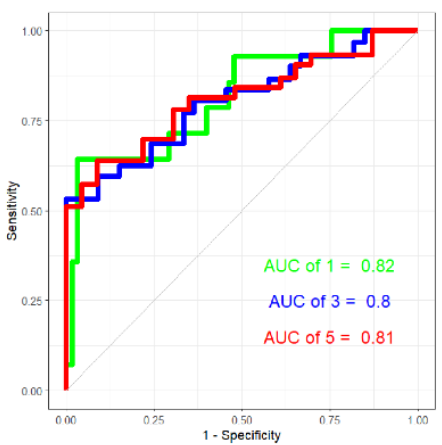

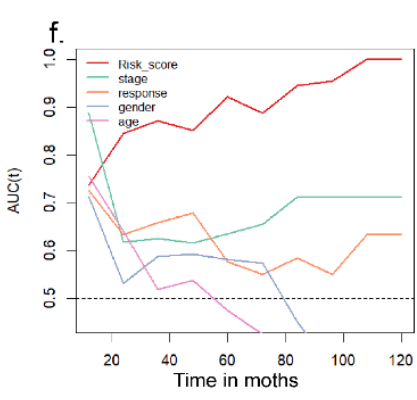

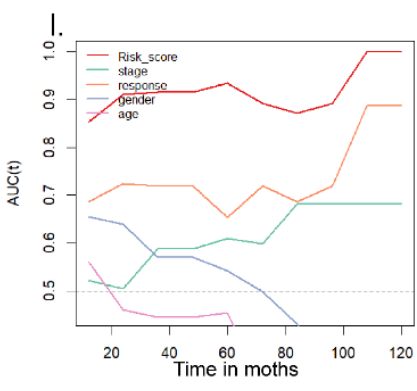

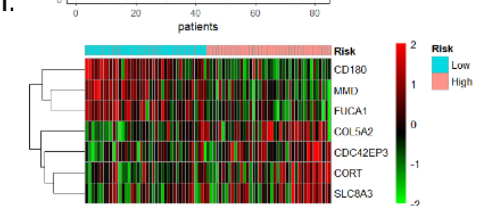


Figure 4. Assessment of the predictive value of the gene signature in the TARGET-OS cohort. (a, f) Distribution of Risk Score predicted using gene signature in high- and low-risk group; OS status (b) and EFS status (h) between the high- and low-risk group; (c, i) Heatmap of the expression profile of the gene signature. Kaplan-Meier survival curve of OS (d) and EFS (j) between the low- and high-risk group; Time-dependent ROC curves for predicting death (e) and relapse (k) at 1, 3, and 5 years. tROC analysis showed that the Risk Score calculated by this signature was more accurate for OS (f) and EFS (l) prediction than other clinicopathological characteristics. (OS: overall survival; EFS: event-free survival)

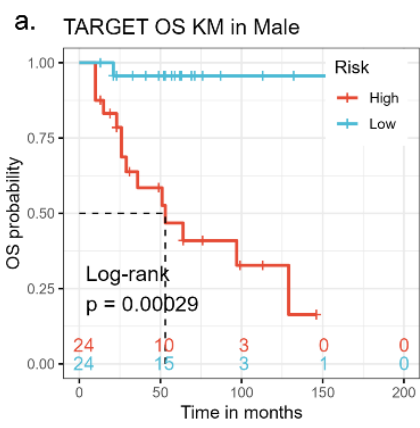

e. TARGET OS KM in Female
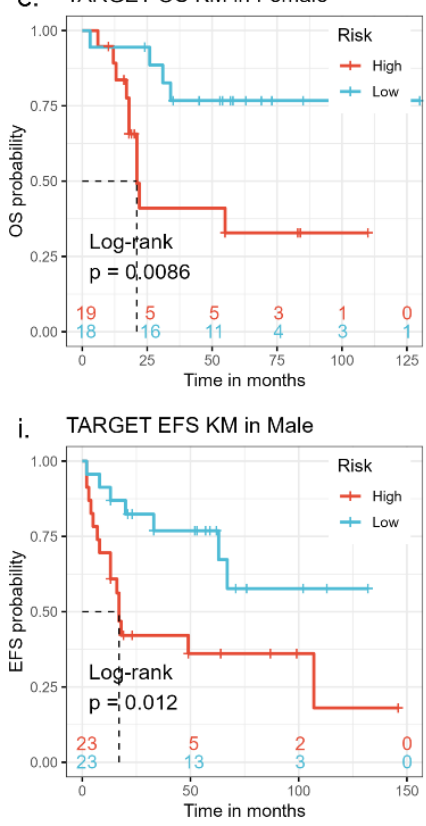

m. TARGET EFS KM in Female

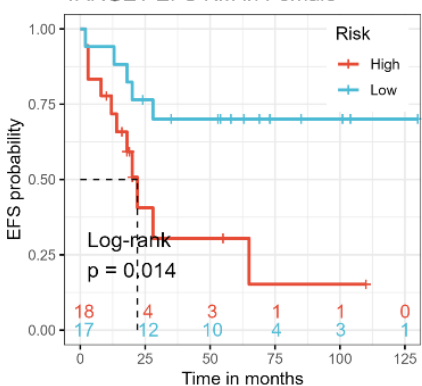

b. TARGET OS KM in stage III

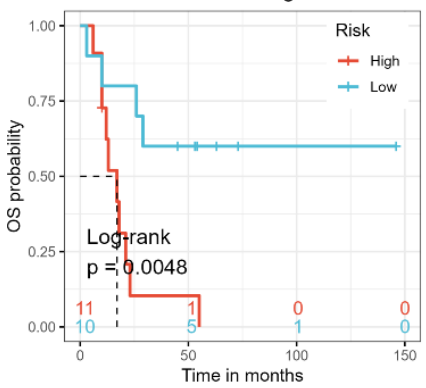

f. TARGET OS KM in stage non-III

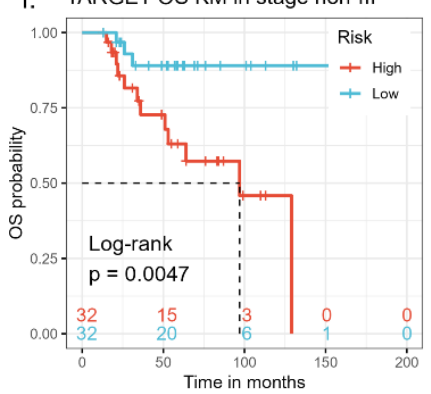

j. TARGET EFS KM in stage III

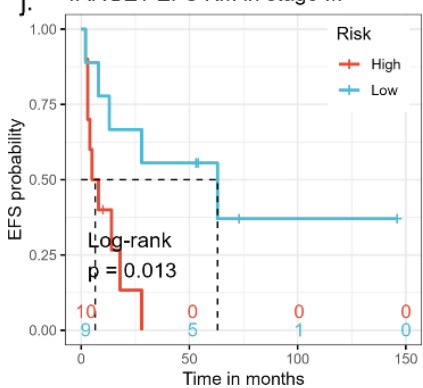

n. TARGET EFS KM in stage non-III

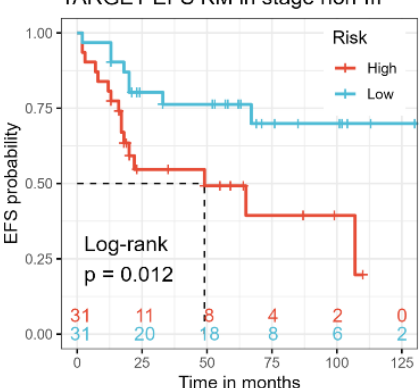

c. TARGET OS KM in adults

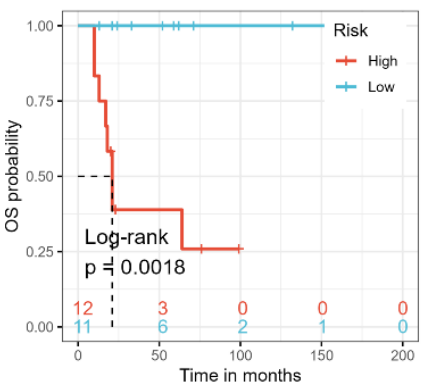

g. TARGET OS KM in minors

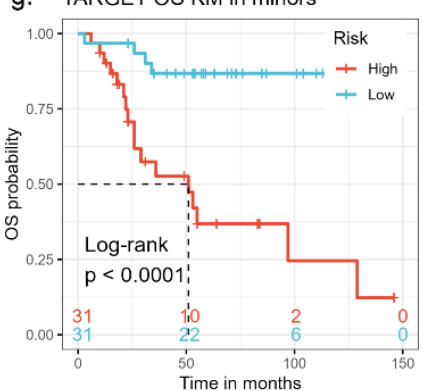

k. TARGET EFS KM in adults

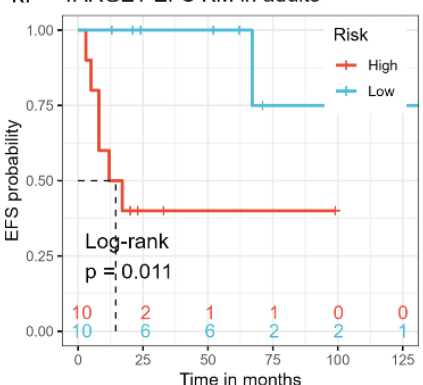

o. TARGET EFS KM in minors

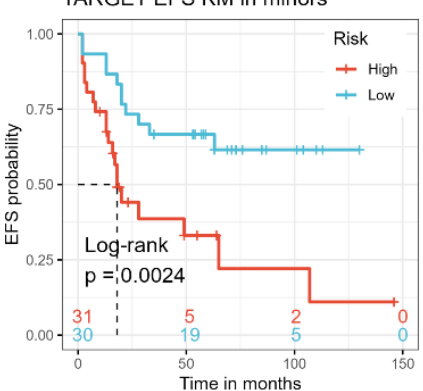

d. TARGET OS KM in huvos I/II

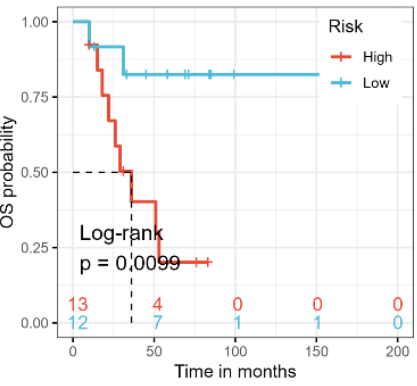

h. TARGET OS KM in huvos III/IV

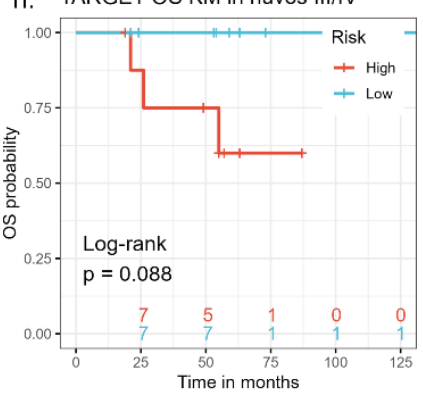

I. TARGET EFS KM in huvos I/II

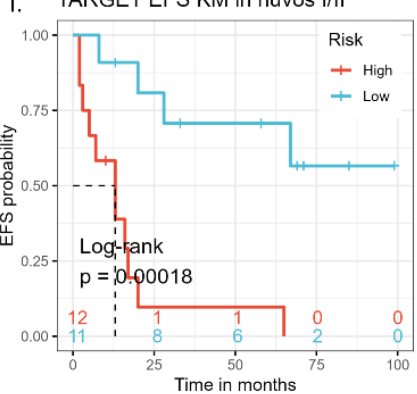

p. TARGET EFS KM in huvos III/IV

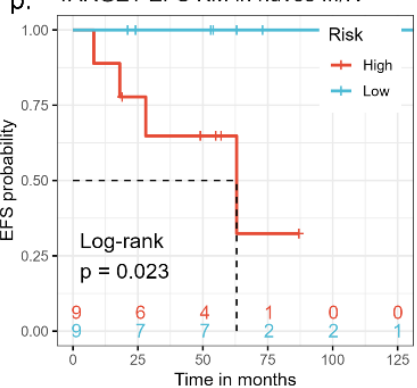

Figure 5. Kaplan-Meier curves for OS and EFS of patients with OSA in different clinical subgroups. (a, e, i, m) KaplanMeier curves of OSA patients in different gender; $(b, f, j, n)$ Kaplan-Meier curves of OSA patients in different stages groups; $(c, g, k, o)$ Kaplan-Meier curves of OSA patients in different age groups; (d, h, l, p) Kaplan-Meier curves of OSA patients in different Huvos grade groups. (OS: overall survival; EFS: event-free survival; OSA: osteosarcoma) 
a.
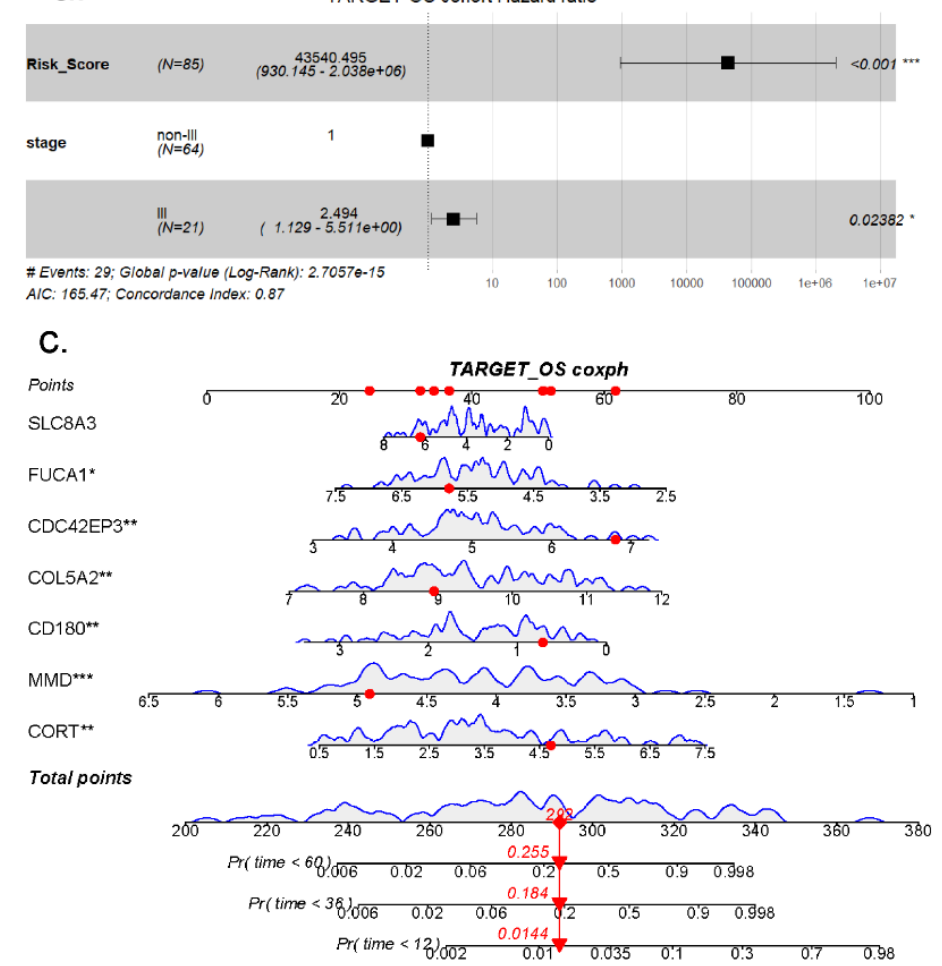

b.
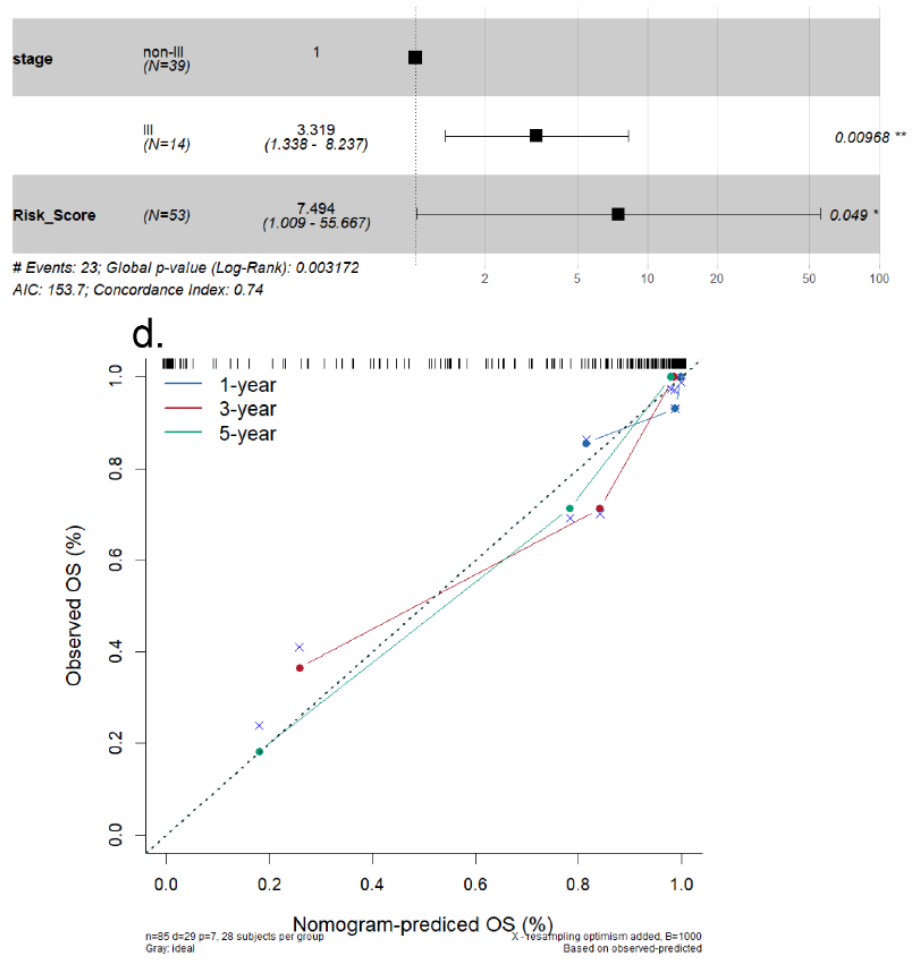

Figure 6. Construction and internal validation of the nomogram in the TARGET-OS cohort. Forrest plot of the multivariate Cox regression analysis using Risk Score and Enneking stage in TARGET-OS cohort (a) and GSE21257 cohort (b); (c) The nomogram based on the 7-gene signature to predict the 1-, 3-, and 5-year overall survival risk of OSA patients; (d) The calibration curve for internal validation of the nomogram for evaluating the overall survival at 1, 3, and 5 years.

\subsection{Identification of Therapeutic Targets for Patients in the High-Risk Group}

We used the Robust Rank Aggregation (RRA) method to integrate three cohorts with a minimal bias to determine the DEGs between low- and high-risk groups stratified by the median Risk Score in each cohort, respectively. A total of 202 robust DEGs were selected, including 139 down-regulated and 63 upregulated genes (Supplementary Table $\mathrm{S1}$ ). According to the robust DEGs' log2 (fold change), we displayed the top 30 upregulated and downregulated ones via heatmap (Figure 7a). Via pathway and process enrichment analyses, the down-regulated genes were found mainly involved with humoral immune response, and the upregulated ones were found involved with extracellular matrix and endochondral ossification (Figure 7b). The downregulated genes were separated into eight modules via protein-protein interaction enrichment analysis and the MCODE algorithm. The top 3 with their corresponding function description are displayed in Figure 7c. In Figure 7d, only two modules and interrelated functions of upregulated genes are shown, illustrating that the genes in MCODE_1 were involved with collagen trimer and protein digestion and absorption. The genes in MCODE_2 were involved with the translation and peptide biosynthetic process. The results above indicated the widespread relevance between this Risk Score and immune status and the possibility of the down-regulated gene perhaps mainly expressed in the immune cells infiltrated in OSA. To testify these two hypotheses, firstly, the correlation analysis between Risk Score and TumorPurity, ImmuneScore, and StromalScore was conducted and displayed in Figure 7e. Secondly, a heatmap (Figure 7f) was drawn to show the expression profiler of the gene within the top 5 modules in different samples, including OSA biopsy specimens (Biopsy), mesenchymal stem cell (MSC), osteoblast (OB), and OSA cell lines (OS), demonstrating many downregulated genes, such as HLA-DRB1, CD33, IFI30, VAMP8 and so on, highly expressed in biopsy specimens, but nearly did not express in OS. Most of the down-regulated genes did not influence the overall survival in the TARGET-OS cohort. Therefore, we reckon 
that the downregulated genes perhaps represent the down-infiltrated immune cells. Therefore, ImmuneCellAI was used to estimate the composition of 24 immune cells in OSA biopsy specimens of the TARGET-OS cohort. Figure $7 \mathrm{~g}$ showed the down-regulated genes indeed positively correlated with many immune cells including, nature regulatory T cell (nTreg), macrophage, natural killer cell (N.K.), Cytotoxic Tcell (Cytotoxic), Type I regulatory T cell (Tr1), Dendritic cells (D.C.), and Gamma delta T cell. Six upregulated genes were identified as risk factors via univariate Cox regression (Figure 7h). Finally, only RPS28 was identified as an independent risk factor via multivariate Cox regression (Figure 7i).
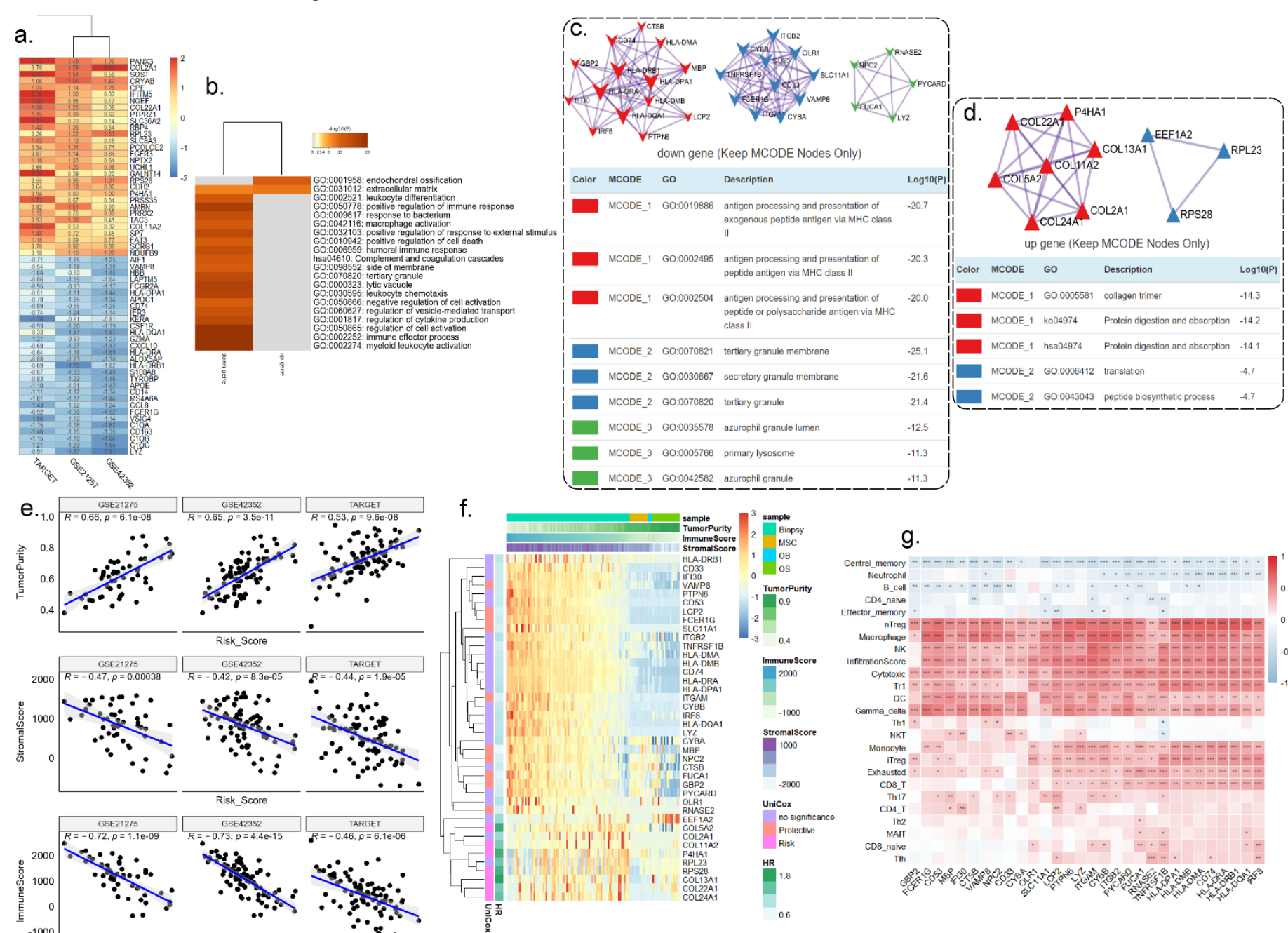

g.

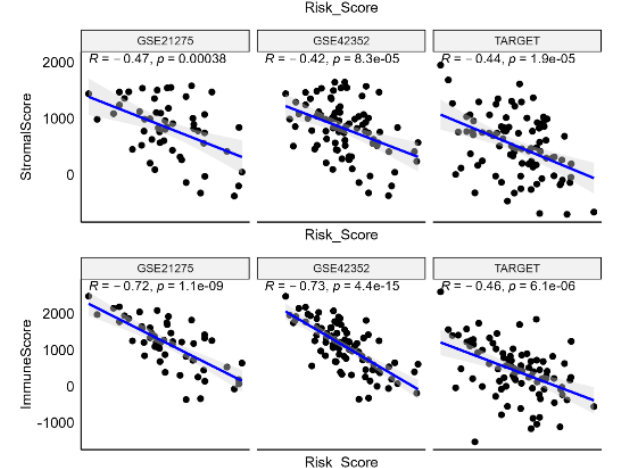

i.

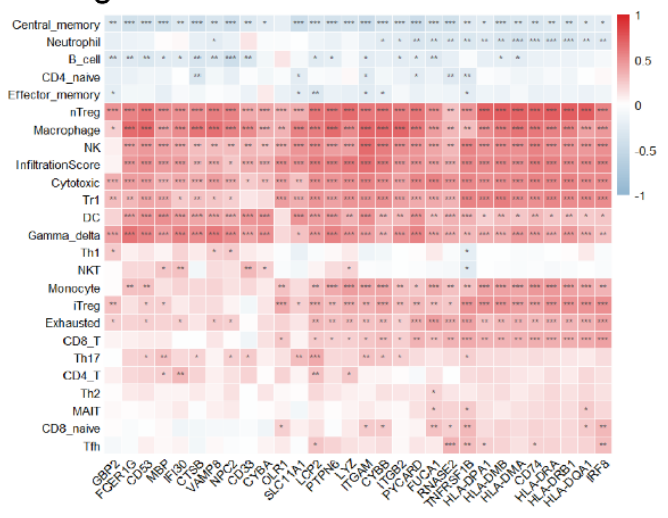

h.

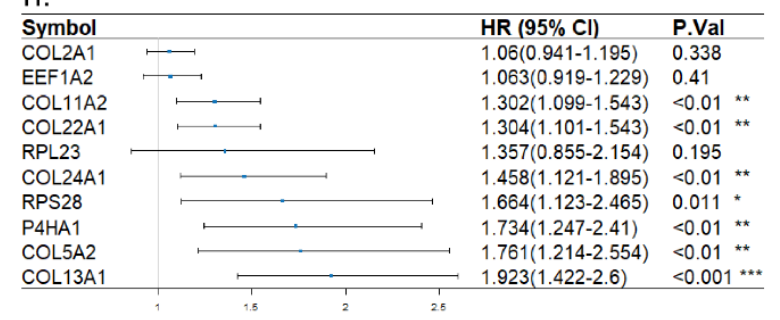

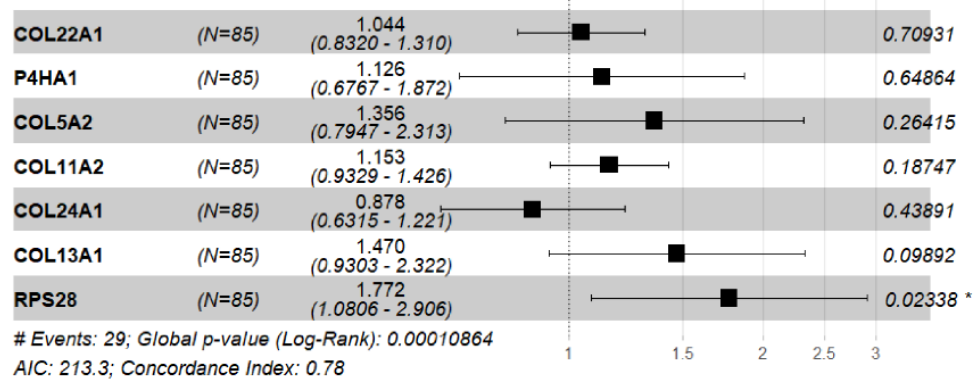

Figure 7. Identification of therapeutic targets for patients in the high-risk group. (a) The heatmap of top 30 upregulated and down-regulated robust DEGs identified by the RRA method. Red represents high-regulated DEGs, while blue represents low-regulated DEGs; (b) The heatmap of enriched top 20 terms across input gene lists, colored by p-value; (c) Proteinprotein interaction network and the top 3 MCODE components identified in the down-regulated gene lists, as well as their corresponding function description. (d) The only two modules and corresponding functions of upregulated genes; (e) 
Scatter plots showing the correlation between Risk Score and TumorPurity, ImmuneScore, and StromalScore across the three cohorts. (f) In the GSE42352 dataset, the heatmap shows the expression profiler of the genes in the top $5 \mathrm{MCODE}$ modules. The samples were grouped according to the sample type and sorted in reverse order of ImmuneScore. (g) The heatmap shows the correlation between 24 immune cells and the down-regulated genes. $(\mathrm{h})$ Forest plot showing the univariate Cox regression analysis of the ten clustered upregulated genes; (c) Forest plot of the multivariate Cox regression analysis showed that RPS28 is an independent risk factor for overall survival in the TARGET-OS cohort. $\left({ }^{*} \mathrm{p}<0.05,{ }^{* *} \mathrm{p}<\right.$ $\left.0.01,{ }^{* * *} \mathrm{p}<0.001\right)$.

3.5 Preliminary Validation of RPS28 as a Promising Therapeutic Target Using Multiple Methods

KM curve showed that the patients with higher RPS28 expression were with much worse OS and EFS in the three cohorts, including TARGET-OS (Figure 8a), GSE21257 (Figure 8b), and GSE42352 (analyzed in R2) (Figure 8c). Figure 8d showed that RPS28 was significantly highly expressed in OSA cell lines compared with the putative progenitor cells of OSA, including MSC and OB. Figure 8e showed RPS28 was significantly highly expressed in OSA specimens when compared with normal bone tissues. Therefore, we reckon that RPS28 may be a promising therapeutic target in OSA cells, not the microenvironment. Nevertheless, it was reported that RPS28 controls MHC class I peptide generation and influences $\mathrm{CD} 8^{+} \mathrm{T}$ cell cancer immunosurveillance [33]. Indeed, in The Human Protein Atlas, RPS28 protein was highly expressed in one of the OSA cell lines, U2-OS, but HAL-A was no staining, supporting the expression of RPS28 and HAL-A may be negatively correlated (Figure 8f). Additionally, across the three cohorts, using the median of RPS28 expression value, patients were separated into the low-RPS28 group and highRPS28 group, and ImmuneScores were found significantly lower in the latter (Figure 8g). By using the Query Gene Function on TIDE (http://tide.dfci.harvard.edu/query/) [34], the expression of RPS28 was found negatively correlated with the cytotoxic T lymphocyte (CTL) level in GSE21257 (Figure 8h).

To further validate the clinical significance of RPS28 in OSA, immunohistochemical staining of RPS28 protein in and around the tumor was performed in the five patients of our hospital, whose tumor necrosis rates were all less than $90 \%$ (Table s1), showing that RPS28 protein was intensively expressed in OSA tissues, but rarely expressed in either para-tumor soft tissue or normal bone tissue (Figure 9a and Figure S3a-c). It was reported that decreased RPS28 protein concentrations impaired Hela cell viability [35]. Therefore, we guessed that RPS28 might promote tumor immune escaping and involve OSA development directly, which was further validated in vitro.

In multiple OSA cell lines, MG-63 showed the highest expression of RPS28 mRNA (Figure 9b), which was selected for further RNA interference experiments, in which siRPS28-3 showed the best interference effect (Figure 9c), whose knockdown efficiency was verified by western blotting analysis (Figure 9d). As shown in Figure 9e-g, the CCK-8 and colony formation assays demonstrated that RPS28 knockdown significantly inhibited the proliferation of MG-63. As mentioned above, the patients with higher RPS28 expression had much worse metastasis-free survival (Figure 8c), indicating that RPS28 may be closely associated with OSA metastasis, which was also validated by wound healing assay, transwell migration, and matrigel invasion assays (Figure 9h-k). 


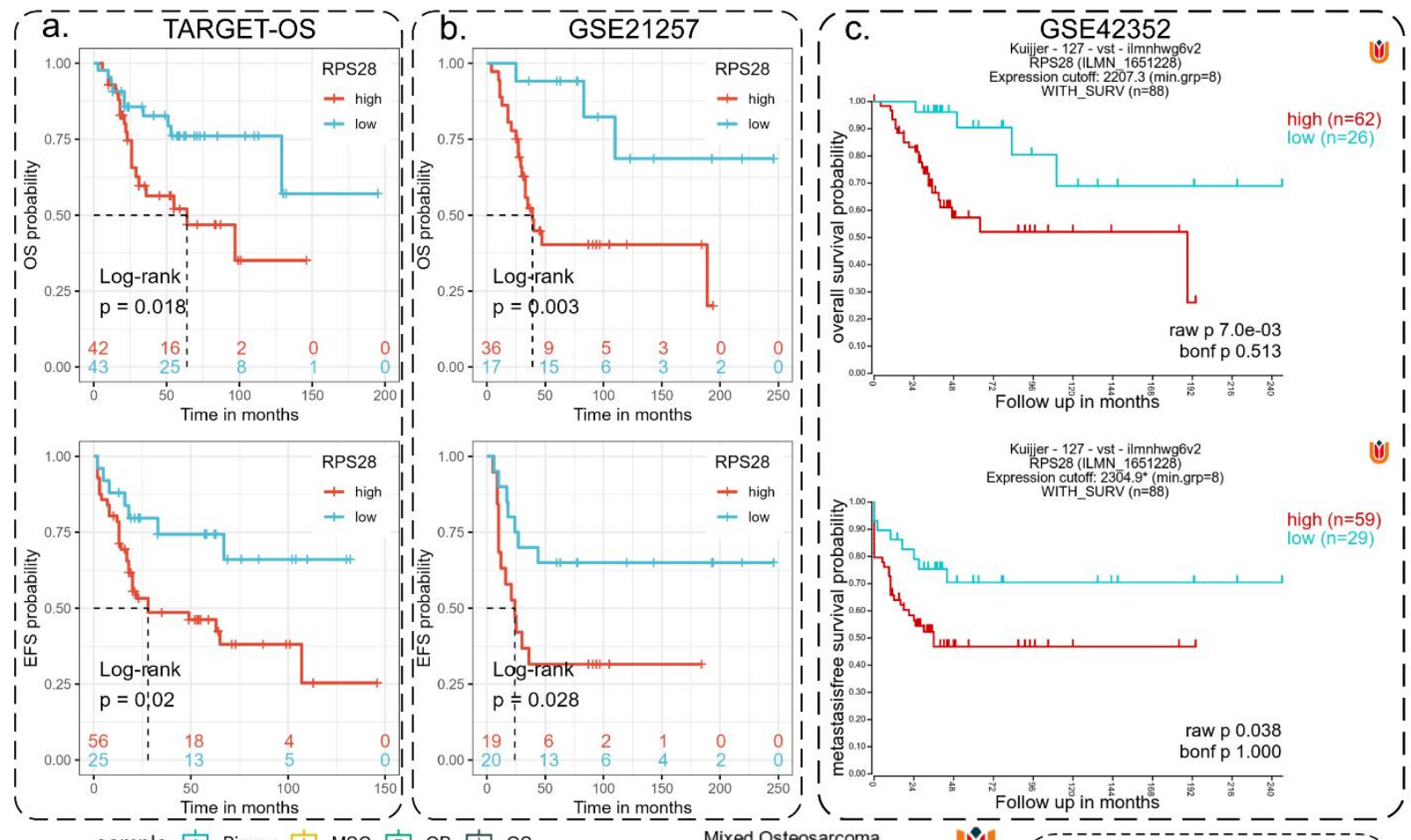

d.
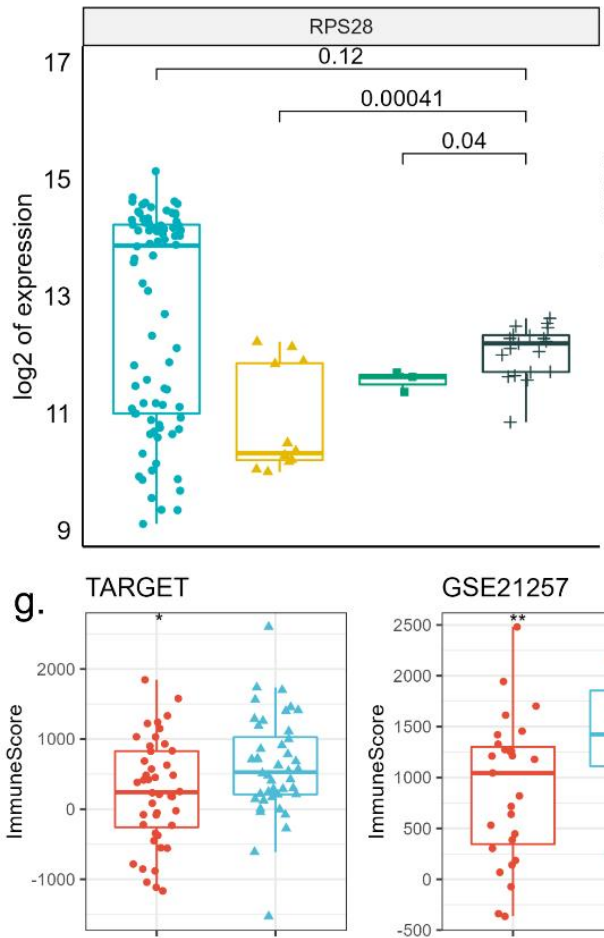

GSE21257

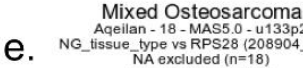
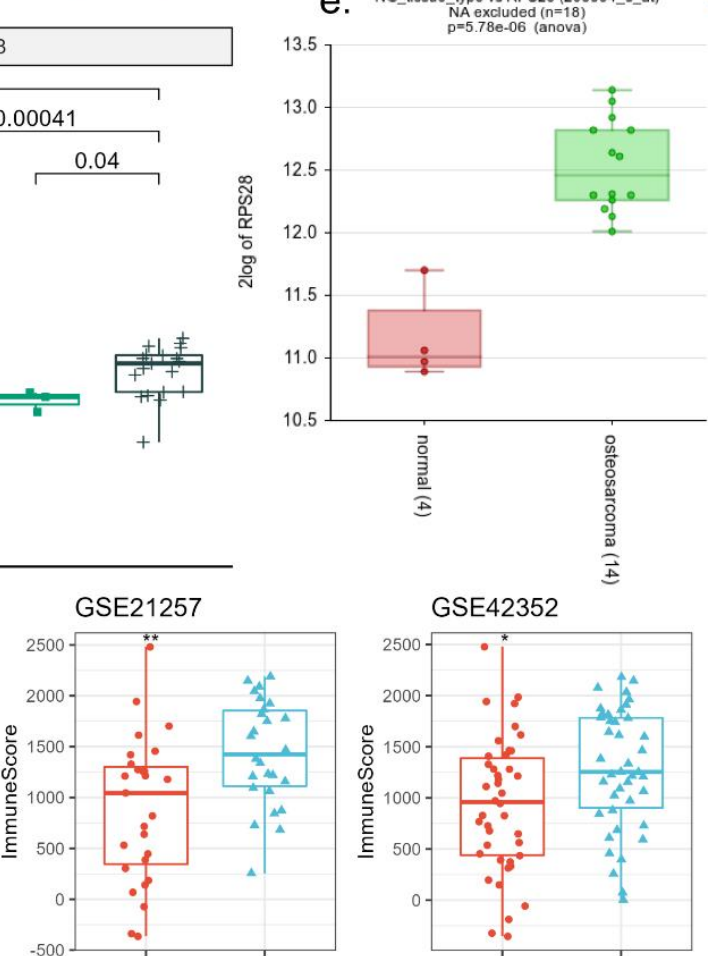

GSE42352

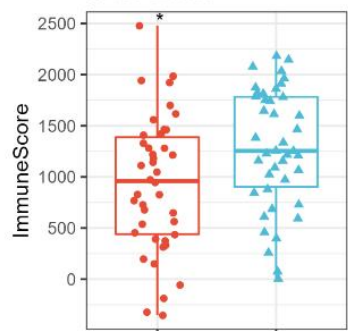

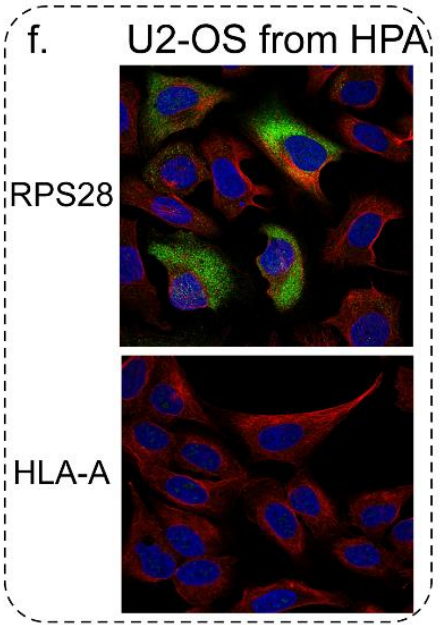

h. $\quad r=-0.439, p=0.00102$

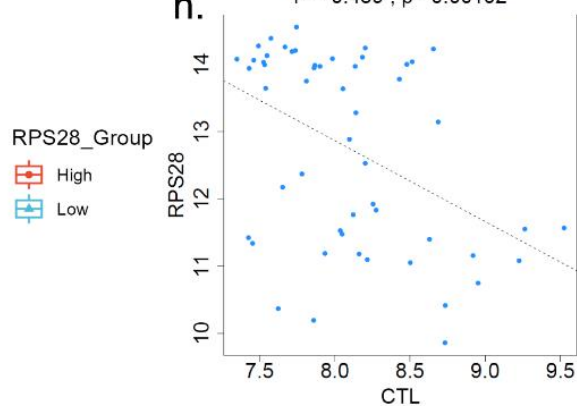

Figure 8. Preliminary validation of RPS28 as a promising therapeutic target in multiple platforms. (a-c) The Kaplan-Meier survival curve of overall survival rate and event-free survival rate among OSA patients between the low-RPS28 group and high-RPS28 group in three cohorts; (d) The expression of RPS28 in different types of samples. (Biopsy: OSA biopsy specimens; MSC: mesenchymal stem cell; O.B.: osteoblast; OS: OSA cell lines); (e) The mRNA expression of RPS28 in OSA specimens and normal bone tissue; (f) Immunofluorescent staining in OSA cell line, U2-OS, showing that RPS28 protein intensively expressed in the cytosol, whereas HLA-A protein was no staining; (g) Boxplots of ImmuneScore in low- and high-RPS28 group across the three cohorts; (h) Scatter plots showing the correlation between the expression of RPS28 with cytotoxic T lymphocyte (CTL) level in GSE21257. 


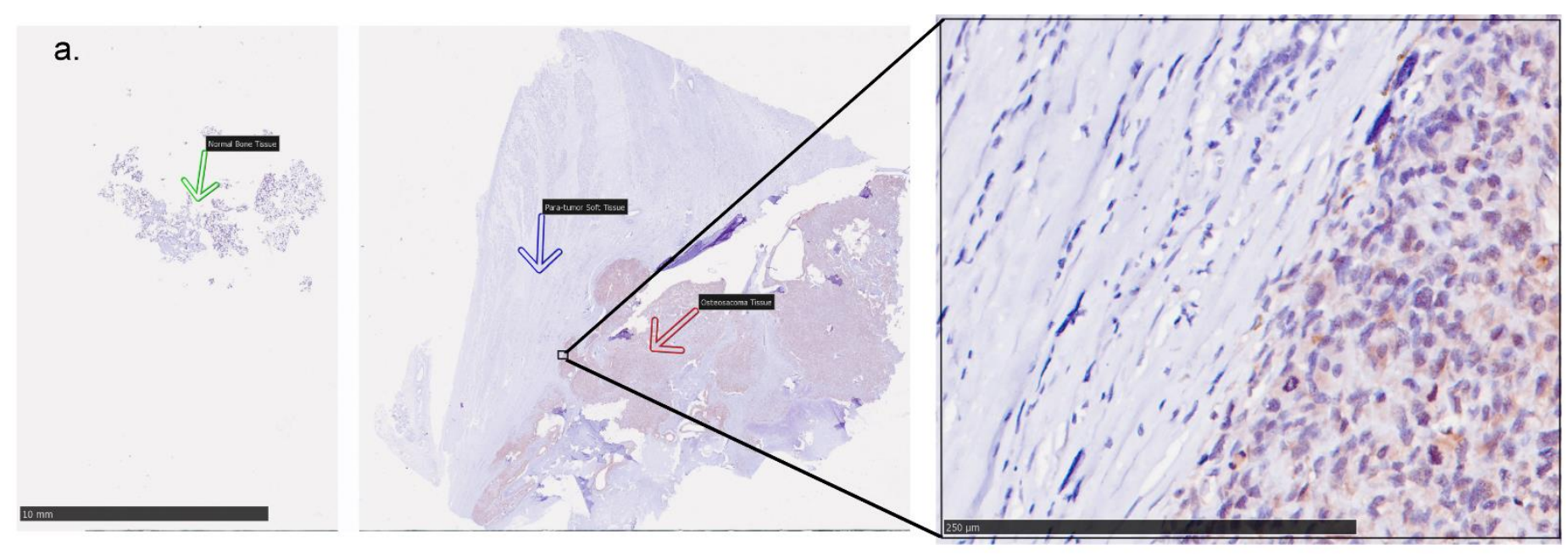

b. RPS28 mRNA

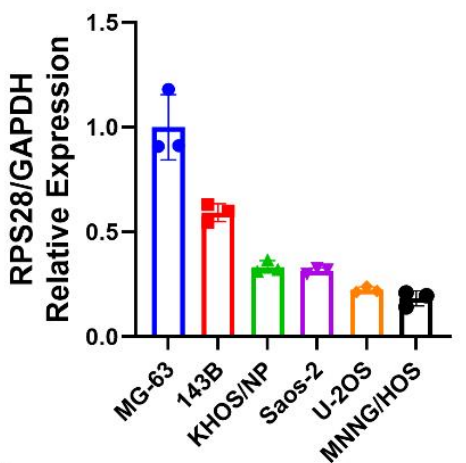

e.
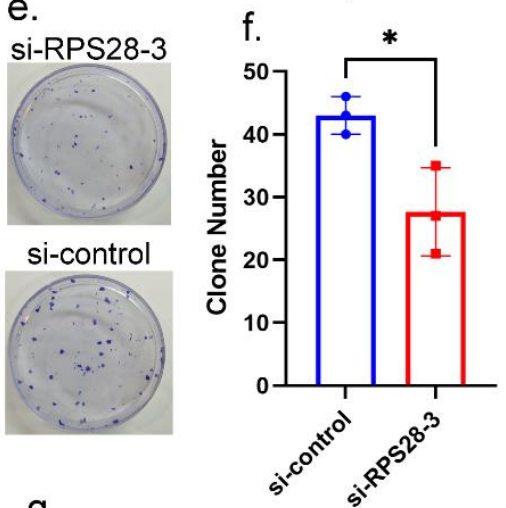

g.

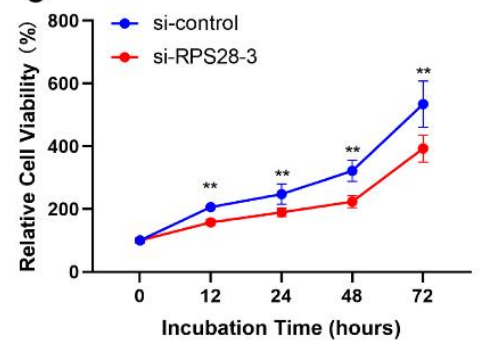

C. RPS28 mRNA

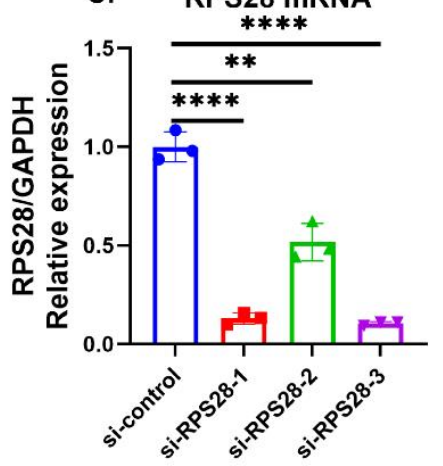

h. si-control
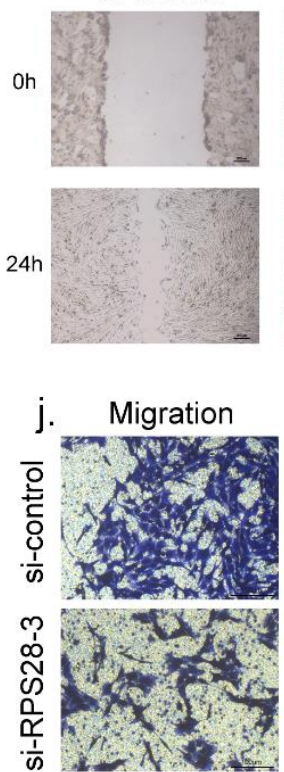

si-RPS28-3
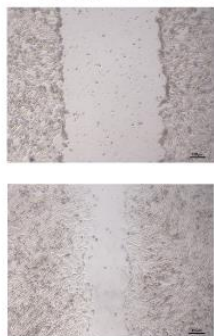

Invasion

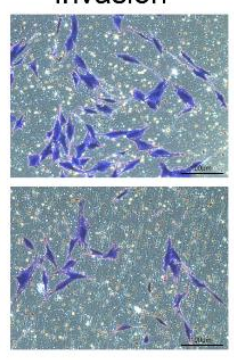

d.

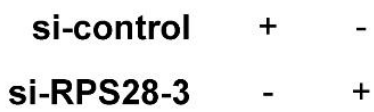

Anti-RPS28

Anti-GAPDH
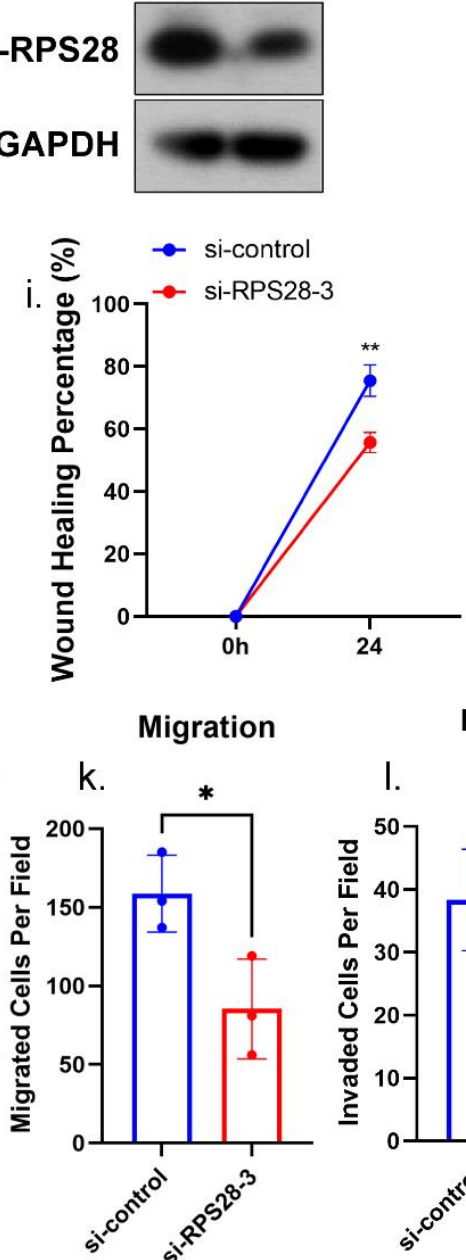

Invasion
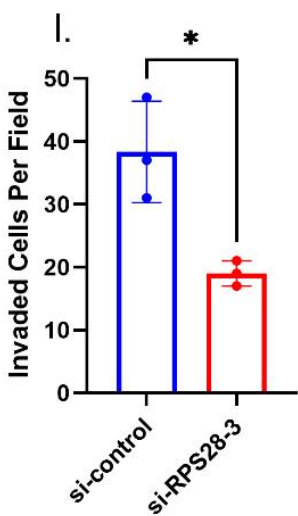

Figure 9. Preliminary validation of RPS28 as a promising therapeutic target by multiple experiments. (a) Immunochemistry staining image of RPS28 in OSA tissue (red arrow), para-tumor soft tissue (blue arrow), and normal bone tissue (green arrow); (b) Relative mRNA expression of RPS28 in human OSA cell lines. (c) Relative mRNA expression of RPS28 in MG63 cell lines after transfection of si-control, si-RPS28-1, si-RPS28-2, si-RPS28-3. (d) Western blotting analysis of the expression of RPS28 in MG-63 cell lines after transfection of si-control and si-RPS28-3. Colony formation assays (e, f) and CCK-8 
(g) were used to assess the proliferation of MG63 cell lines. (h, i) Migration ability was evaluated by scratch assay, and the wound healing percentage was calculated $(n=3) .(j, k, 1)$ Migration and invasion abilities were assessed by transwell assay with or without Matrigel layer. $\left({ }^{*} \mathrm{p}<0.05,{ }^{* *} \mathrm{p}<0.01,{ }^{* * *} \mathrm{p}<0.001,{ }^{* * *} \mathrm{p}<0.0001\right)$

\section{Discussion}

With the establishment of standard therapy for OSA in the 1980 s, $60 \%$ of patients with localized diseases achieved long-term survival [4]. Nevertheless, limited therapeutic progress has been made since that time. We reckon it is essential to recognize the highrisk patients who might not respond to standard therapy and develop supplementary treatments for these patients.

Despite being the most common primary bone cancer in children and young adults, OSA remains a rare but genomically complex malignancy [36]. As a result, it is challenging to get enough OSA specimens in a short time when we want to start research. Therefore, it is crucial to conduct big-data analysis by integrating multiple public datasets for discovering universal and robust therapeutic targets.

In the present research, we firstly identified six hallmark gene sets that were involved with the overall survival of OSA patients, in which UV RESPONSE UP containing the genes upregulated in response to ultraviolet (U.V.) radiation, and CHOLESTEROL HOMEOSTASIS containing the genes involved in cholesterol homeostasis were found as risk factors. In contrast, INTERFERON GAMMA RESPONSE containing the genes upregulated in response to IFNG, ALLOGRAFT REJECTION containing the genes upregulated during transplant rejection, IL6 JAK STAT3 SIGNALING containing the genes upregulated by IL6 via STAT3, and XENOBIOTIC METABOLISM containing the genes encoding proteins involved in the processing of drugs and other xenobiotics were found as protective factors. Subsequently, a multivariate Cox model was conducted based on hallmark gene sets, including UV RESPONSE UP, CHOLESTEROL HOMEOSTASIS, and XENOBIOTIC METABOLISM. However, because the microarray datasets lack a lot of genes, the results of ssGSEA across the three cohorts were not robust enough. As a result, the predictive value of this model could not be validated in independent cohorts (the data were not displayed). But we still reckon it is an important discovery. Because it was reported that disturbed cholesterol balance underlies not only cardiovascular disease but also an increasing number of other diseases such as neurodegenerative diseases and cancers [37], and we revealed that cholesterol homeostasis might also influence the overall survival of OSA patients, which is worth studying intensely. For constructing a universal model across the three cohorts, a seven-gene signature composed of CORT, MMD, CD180, COL5A2, CDC42EP3, FUCA1, and SLC8A3 was established.

Monocyte to macrophage differentiation-associated (MMD) protein is a member of the progestin and AdipoQ receptor (PAQR) family and was found in mature macrophages and reported to be associated with macrophage activation in vitro [38]. CD180, also known as RP105, is a cell surface molecule consisting of extracellular leucine-rich repeats (LLR) and a short cytoplasmic tail. The extracellular LRR is associated with a molecule called MD-1 and forms the cell surface receptor complex, RP105/MD-1. It belongs to the family of pathogen receptors, Toll-like receptors (TLR). RP105/MD1 controls B cell recognition and lipopolysaccharide (LPS) signaling by working with TLR4 [39]. Solute carrier family 8 member A3 (SLC8A3) encodes a member of the sodium/calcium exchanger integral membrane protein family. The downregulation of SLC8A3 is positively correlated with the prognosis of stage II-IV colon cancer [40]. Interestingly, human macrophages and monocytes express functional SLC8A3 [41]. Cortistatin (CORT) encodes a neuropeptide that shares high homology with somatostatin (somatotropin release-inhibiting factor, SRIF) and can bind with high affinity to all somatostatin (SST) receptor subtypes [42]. Although a report has shown that CORT could inhibit the proliferation in cancer cells [43], it is a risk factor in OSA, according to the results of this study. Because the transcriptome data were from bulk RNA-sequencing, the mRNA detected came from OSA cells and infiltrating immune and stromal cells. As a result, the contradiction is understandable. 
Precisely, CORT could be detected in cells and tissues from the human immune system and played an essential role in monocyte differentiation and activation [44]. Collagen type $\mathrm{V}$ alpha 2 chain (COL5A2) encodes an alpha chain for one of the low abundance fibrillar collagens, which is the component of the extracellular matrix and is an unfavorable prognostic factor in gastric cancer [45] and bladder cancer [46]. CDC42 effector protein 3 (CDC42EP3) is a member of the cell division cycle 42 (CDC42) effector protein family. Previous studies showed that CDC42EP3 plays a role in the function of cancer-associated fibroblasts and DNA damage response [47-49] and is a promoter in gastric cancer and colorectal cancer [50,51]. The protein encoded by Alpha-L-fucosidase 1 (FUCA1) is a lysosomal enzyme involved in the degradation of fucose-containing glycoproteins and glycolipids. It was reported that FUCA1 as a target of p53 functions as a tumor suppressor in colon cancer [52], luminal B LN+ breast cancer [53], and thyroid cancer [54], but as a tumor promoter in glioma instead [55]. Tumor suppressor protein p53 encoded by Tumor protein p53 (TP53) is a tumor suppressor associated with OSA [56]. It is believable that FUCA1, one of the targets of p53, functions as a protective factor in OSA. As mentioned above, this seven-gene signature is reasonable, which was further validated in both internal and external OSA cohorts.

Using this seven-gene signature, the Risk Score of each case was calculated, whose median was used to separate patients into high- and low-risk groups. KM survival analysis demonstrated that patients in the high-risk group have much poor overall and eventfree survival in the training and validation cohorts. To further estimate the predictive value of this signature, KM survival analysis was conducted in several clinical sub-groups in the TARGET-OS cohort. Consistently, in all clinical subgroups, patients in the high-risk group had poorer overall and event-free survival except for in the huvos III/IV, which is because the cases in this sub-group were too little to get a statistical significance. Timedependent ROC curve analysis confirmed the satisfactory accuracy of this predictive model in 1-, 3- and 5- years. Compared with clinicopathological characteristics, the predictive value of this signature exhibited the most potent and stable ability for predicting overall and evet-free survival most of the time. Additionally, multivariate Cox regression analysis showed that both Risk Score and Enneking stage were independent risk factors in both the TARGET-OS cohort and GSE21257 cohort. Taken together, the results mentioned above proved this seven-gene signature had a substantial predictive value in OSA patients. A nomogram composed of the signature was designed to facilitate stratifying patients based on the TPM value of seven genes obtained from the bulk RNA sequencing using their biopsy specimens.

To discover effective therapeutic targets for high-risk OSA patients, we firstly identified the differentially expressed gene between high- and low-risk patients in the TARGET-OS cohort, GSE21257 cohort, and GSE42352 cohort by using Differential Expression Analysis and RRA algorithms. Then, by constructing the PPI network and identifying the highly interconnected genes using the MCODE algorithm, the top 3 functional subnet modules in down-regulated genes and the only two functional subnet modules in upregulated genes were identified used for further analyses. According to the results of G.O. enrichment analyses, most down-regulated genes were enriched in immune-related biological processes and cellular components, including antigen processing and presentation of peptide antigen via MHC class II, tertiary granule, and primary lysosome. Further results showed that Risk Score was negatively correlated with ImmuneScore, and most of the down-regulated genes almost did not express in OSA cell lines, indicating they might represent several immune cells infiltrating in OSA specimens, which could be a potential therapeutic target in the tumor microenvironment. The only two MCODES in the upregulated genes were enriched in collagen trimer and translation, in which prolyl 4-hydroxylase subunit alpha 1 (P4HA1) was validated as a risk factor, promoting the metastasis of OSA cells in vitro and in vivo [15]. However, RPS28 was finally found as an independent risk factor, which was validated further by multiple methods, including KM survival analyses, comparing of RPS28 expression between OSA 
tissues and normal tissues in mRNA level and protein level, RNA interference experiment, and so on.

Ribosomes, consisting of a small $40 \mathrm{~S}$ subunit and a large $60 \mathrm{~S}$ subunit, are responsible for translating information contained in mRNA into functional proteins, the ultimate step in the genetic program [57]. Notably, the hyperactivation of ribosome biogenesis, which can be initiated by oncogenes or the loss of tumor suppressor genes, had a critical role in cancer initiation and progression [58]. Ribosomal protein S28 (RPS28) gene, located at 19p13.2, encodes a ribosomal protein that is a component of the small $40 \mathrm{~S}$ subunit. A report showed that RPS28 controls MHC class I peptide generation and influences CD8 ${ }^{+} \mathrm{T}$ cell cancer immunosurveillance in melanoma [33]. We reckon that RPS28 might play the same role in OSA as a risk factor, based on the pieces of evidence including U2OS cell lines with an intensive expression of RPS28 protein but no expression of HLA-A, the OSA patients in the high-RPS28 group with lower ImmuneScores, and the negative correlation between the expression of RPS28 mRNA with CTL level in OSA cohort GSE21257. Additionally, in vitro experiments showed that RPS28 knockdown impaired the proliferation, migration, and invasion of MG-63 cell lines, consistent with its influence exerted in Hela cell lines [35] and three OSA cohorts mentioned above. Our results suggest that RPS28 functions as a tumor promoter in OSA by promoting the abilities of proliferation and metastasis of tumor cells, simultaneously facilitating immune escape, making it a potential therapeutic target worthy of further investigation.

Some limitations still existed. Firstly, the size of used cohorts was relatively small, and some cases had missing clinical information. Consequently, we are constructing a more extensive multi-omics OSA database using our cohort. Secondly, the detailed mechanisms underlying how RPS28 exerts its effects mentioned above and the exact efficacy of RPS28 depletion in vivo require further exploration.

\section{Conclusion}

In conclusion, by multiple methods, we identified a seven-gene prognostic signature for OSA patients and a calculated Risk Score that was a robust independent prognostic biomarker. Additionally, according to the signature, a nomogram was conducted to predict the prognosis and stratification for OSA patients based on the TPM value of the seven genes obtained from the bulk RNA sequencing using their biopsy specimens. Finally, We found that RPS28 was a promising therapeutic target for the patients in the high-risk group separated by the signature.

Supplementary Materials: The following are available online at www.mdpi.com/xxx/s1, Figure S1. WGCNA analysis in TARGET-OS cohort, Figure S2. External validation of the predictive value of the gene signature, Figure S3. Immunochemistry staining of RPS28 in our cohort, Table S1. Differentially expressed genes (DEGs) between high- and low-risk groups with $\mid \log 2$ (fold change) $\mid>0.69$ and $\mathrm{p}$-Value $<0.05$ by RRA analysis.

\section{Author Contributions:}

Funding: This research was funded by the National Nature Science Founding of China (82072962).

Institutional Review Board Statement: The study was conducted according to the guidelines of the Declaration of Helsinki and approved by the Institutional Review Board (2020-YS-060).

Informed Consent Statement: The databases used in this study are all openly available, in which the patients involved have obtained ethical approval. Users can download relevant data for free for research and publish relevant articles.

Data Availability Statement: The data presented in this study are openly available in Genomic Data Commons (GDC) Data Portal, Gene Expression Omnibus (GEO), R2: Genomics Analysis, and Visualization Platform, The Human Protein Atlas (HPA).

Acknowledgments: The authors would like to thank TARGET, GEO, R2, the HPA, and TIDE for providing their platforms, contributors for uploading their meaningful datasets, Dr. Jianming Zeng 
(University of Macau), Xiaojie Sun, and all members of their bioinformatics team, biotrainee, for generously sharing their experience and code.

Conflicts of Interest: The authors declare no competing financial and non-financial conflicts of interest.

\section{References}

1. Mirabello, L.; Troisi, R.J.; Savage, S.A. Osteosarcoma incidence and survival rates from 1973 to 2004 : data from the Surveillance, Epidemiology, and End Results Program. Cancer 2009, 115, 1531-1543, doi:10.1002/cncr.24121.

2. Link, M.P.; Goorin, A.M.; Horowitz, M.; Meyer, W.H.; Belasco, J.; Baker, A.; Ayala, A.; Shuster, J. Adjuvant chemotherapy of high-grade osteosarcoma of the extremity. Updated results of the Multi-Institutional Osteosarcoma Study. Clin. Orthop. Relat. Res. 1991, 8-14.

3. Goorin, A.M.; Schwartzentruber, D.J.; Devidas, M.; Gebhardt, M.C.; Ayala, A.G.; Harris, M.B.; Helman, L.J.; Grier, H.E.; Link, M.P. Presurgical chemotherapy compared with immediate surgery and adjuvant chemotherapy for nonmetastatic osteosarcoma: Pediatric Oncology Group Study POG-8651. J. Clin. Oncol. 2003, 21, 1574-1580, doi:10.1200/jco.2003.08.165.

4. Isakoff, M.S.; Bielack, S.S.; Meltzer, P.; Gorlick, R. Osteosarcoma: Current Treatment and a Collaborative Pathway to Success. J. Clin. Oncol. 2015, 33, 3029-3035, doi:10.1200/JCO.2014.59.4895.

5. Stiller, C.A.; Bielack, S.S.; Jundt, G.; Steliarova-Foucher, E. Bone tumours in European children and adolescents, $1978-1997$. Report from the Automated Childhood Cancer Information System project. Eur. J. Cancer 2006, 42, 2124-2135, doi:10.1016/j.ejca.2006.05.015.

6. Smith, M.A.; Seibel, N.L.; Altekruse, S.F.; Ries, L.A.; Melbert, D.L.; O'Leary, M.; Smith, F.O.; Reaman, G.H. Outcomes for children and adolescents with cancer: challenges for the twenty-first century. J. Clin. Oncol. 2010, 28, 2625-2634, doi:10.1200/jco.2009.27.0421.

7. Steffner, R.J.; Jang, E.S. Staging of Bone and Soft-tissue Sarcomas. J. Am. Acad. Orthop. Surg. 2018, 26, e269-e278, doi:10.5435/JAAOS-D-17-00055.

8. Bacci, G.; Longhi, A.; Versari, M.; Mercuri, M.; Briccoli, A.; Picci, P. Prognostic factors for osteosarcoma of the extremity treated with neoadjuvant chemotherapy: 15-year experience in 789 patients treated at a single institution. Cancer 2006, 106, 1154-1161, doi:10.1002/cncr.21724.

9. Huvos, A.G. Bone tumors: Diagnosis, treatment and prognosis Second edition; WB Saunders CBS Educ and Professional Publ: United States, 1987.

10. Letaief, F.; Khrouf, S.; Yahiaoui, Y.; Hamdi, A.; Gabsi, A.; Ayadi, M.; Mezlini, A. Prognostic factors in High-Grade Localized Osteosarcoma of the Extremities: The Tunisian Experience. J. Orthop. Surg. (Hong Kong) 2020, 28, 2309499020974501, doi:10.1177/2309499020974501.

11. Marina, N.M.; Smeland, S.; Bielack, S.S.; Bernstein, M.; Jovic, G.; Krailo, M.D.; Hook, J.M.; Arndt, C.; van den Berg, H.; Brennan, B.; et al. Comparison of MAPIE versus MAP in patients with a poor response to preoperative chemotherapy for newly diagnosed high-grade osteosarcoma (EURAMOS-1): an open-label, international, randomised controlled trial. The Lancet Oncology 2016, 17, 1396-1408, doi:10.1016/s1470-2045(16)30214-5.

12. Zhu, W.; Xie, L.; Han, J.; Guo, X. The Application of Deep Learning in Cancer Prognosis Prediction. Cancers (Basel) 2020, 12, doi:10.3390/cancers12030603.

13. Deng, Y.; Yuan, W.; Ren, E.; Wu, Z.; Zhang, G.; Xie, Q. A four-methylated LncRNA signature predicts survival of osteosarcoma patients based on machine learning. Genomics 2021, 113, 785-794, doi:10.1016/j.ygeno.2020.10.010.

14. Fu, Y.; Bao, Q.; Liu, Z.; He, G.; Wen, J.; Liu, Q.; Xu, Y.; Jin, Z.; Zhang, W. Development and Validation of a HypoxiaAssociated Prognostic Signature Related to Osteosarcoma Metastasis and Immune Infiltration. Frontiers in Cell and Developmental Biology 2021, 9, doi:10.3389/fcell.2021.633607. 
15. Yang, M.; Ma, X.; Wang, Z.; Zhang, T.; Hua, Y.; Cai, Z. Identification of a novel glycolysis-related gene signature for predicting the prognosis of osteosarcoma patients. Aging (Albany NY) 2021, 13, 12896-12918, doi:10.18632/aging.202958.

16. Chen, Z.; Huang, H.; Wang, Y.; Zhan, F.; Quan, Z. Identification of Immune-Related Genes MSR1 and TLR7 in Relation to Macrophage and Type-2 T-Helper Cells in Osteosarcoma Tumor Micro-Environments as Anti-metastasis Signatures. Frontiers in molecular biosciences 2020, 7, 576298, doi:10.3389/fmolb.2020.576298.

17. Zheng, Y.; Tian, H.; Zhou, Z.; Xiao, C.; Liu, H.; Liu, Y.; Wang, L.; Fan, T.; Zheng, B.; Tan, F.; et al. A Novel Immune-Related Prognostic Model for Response to Immunotherapy and Survival in Patients With Lung Adenocarcinoma. Front Cell Dev Biol 2021, 9, 651406, doi:10.3389/fcell.2021.651406.

18. Li, G.Q.; Wang, Y.K.; Zhou, H.; Jin, L.G.; Wang, C.Y.; Albahde, M.; Wu, Y.; Li, H.Y.; Zhang, W.K.; Li, B.H.; et al. Application of Immune Infiltration Signature and Machine Learning Model in the Differential Diagnosis and Prognosis of Bone-Related Malignancies. Front Cell Dev Biol 2021, 9, 630355, doi:10.3389/fcell.2021.630355.

19. Langfelder, P.; Horvath, S. WGCNA: an R package for weighted correlation network analysis. BMC Bioinformatics 2008, 9 , 559, doi:10.1186/1471-2105-9-559.

20. Su, Z.; Yang, B.; Zeng, Z.; Zhu, S.; Wang, C.; Lei, S.; Jiang, Y.; Lin, L. Metastasis-associated gene MAPK15 promotes the migration and invasion of osteosarcoma cells via the c-Jun/MMPs pathway. Oncol. Lett. 2020, 20, 99-112, doi:10.3892/ol.2020.11544.

21. Tang, X.; Luo, L.; Li, Y.; Wu, H.; Hu, Q.; Yue, H.; He, X.; Zou, J.; Min, S. Therapeutic potential of targeting HSPA5 through dual regulation of two candidate prognostic biomarkers ANXA1 and PSAT1 in osteosarcoma. Aging (Albany NY) 2020, 13, 1212-1235, doi:10.18632/aging.202258.

22. Fan, H.; Lu, S.; Wang, S.; Zhang, S. Identification of critical genes associated with human osteosarcoma metastasis based on integrated gene expression profiling. Mol Med Rep 2019, 20, 915-930, doi:10.3892/mmr.2019.10323.

23. Sun, J.; Zhao, T.; Zhao, D.; Qi, X.; Bao, X.; Shi, R.; Su, C. Development and validation of a hypoxia-related gene signature to predict overall survival in early-stage lung adenocarcinoma patients. Ther. Adv. Med. Oncol. 2020, 12, 1758835920937904, doi:10.1177/1758835920937904.

24. Hanahan, D.; Weinberg, R.A. Hallmarks of cancer: the next generation. Cell 2011, 144, 646-674, doi:10.1016/j.cell.2011.02.013.

25. Liberzon, A.; Birger, C.; Thorvaldsdottir, H.; Ghandi, M.; Mesirov, J.P.; Tamayo, P. The Molecular Signatures Database (MSigDB) hallmark gene set collection. Cell Syst 2015, 1, 417-425, doi:10.1016/j.cels.2015.12.004.

26. Niu, J.; Yan, T.; Guo, W.; Wang, W.; Zhao, Z.; Ren, T.; Huang, Y.; Zhang, H.; Yu, Y.; Liang, X. Identification of Potential Therapeutic Targets and Immune Cell Infiltration Characteristics in Osteosarcoma Using Bioinformatics Strategy. Front. Oncol. 2020, 10, 1628, doi:10.3389/fonc.2020.01628.

27. Zhou, Y.; Zhou, B.; Pache, L.; Chang, M.; Khodabakhshi, A.H.; Tanaseichuk, O.; Benner, C.; Chanda, S.K. Metascape provides a biologist-oriented resource for the analysis of systems-level datasets. Nature Communications 2019, 10, doi:10.1038/s41467-019-09234-6.

28. Vrieze, S.I. Model selection and psychological theory: a discussion of the differences between the Akaike information criterion (AIC) and the Bayesian information criterion (BIC). Psychol. Methods 2012, 17, 228-243, doi:10.1037/a0027127.

29. Kolde, R.; Laur, S.; Adler, P.; Vilo, J. Robust rank aggregation for gene list integration and meta-analysis. Bioinformatics 2012, 28, 573-580, doi:10.1093/bioinformatics/btr709.

30. Yoshihara, K.; Shahmoradgoli, M.; Martinez, E.; Vegesna, R.; Kim, H.; Torres-Garcia, W.; Trevino, V.; Shen, H.; Laird, P.W.; Levine, D.A.; et al. Inferring tumour purity and stromal and immune cell admixture from expression data. Nat Commun 2013, 4, 2612, doi:10.1038/ncomms3612. 
31. Miao, Y.R.; Zhang, Q.; Lei, Q.; Luo, M.; Xie, G.Y.; Wang, H.; Guo, A.Y. ImmuCellAI: A Unique Method for Comprehensive T-Cell Subsets Abundance Prediction and its Application in Cancer Immunotherapy. Adv Sci (Weinh) 2020, 7, 1902880, doi:10.1002/advs.201902880.

32. Wang, Y.; Zeng, L.; Liang, C.; Zan, R.; Ji, W.; Zhang, Z.; Wei, Y.; Tu, S.; Dong, Y. Integrated analysis of transcriptome-wide m(6)A methylome of osteosarcoma stem cells enriched by chemotherapy. Epigenomics 2019, 11, 1693-1715, doi:10.2217/epi2019-0262.

33. Wei, J.; Kishton, R.J.; Angel, M.; Conn, C.S.; Dalla-Venezia, N.; Marcel, V.; Vincent, A.; Catez, F.; Ferre, S.; Ayadi, L.; et al. Ribosomal Proteins Regulate MHC Class I Peptide Generation for Immunosurveillance. Mol. Cell 2019, 73, 1162-1173 e1165, doi:10.1016/j.molcel.2018.12.020.

34. Jiang, P.; Gu, S.; Pan, D.; Fu, J.; Sahu, A.; Hu, X.; Li, Z.; Traugh, N.; Bu, X.; Li, B.; et al. Signatures of T cell dysfunction and exclusion predict cancer immunotherapy response. Nat. Med. 2018, 24, 1550-1558, doi:10.1038/s41591-018-0136-1.

35. Kim, H.K.; Fuchs, G.; Wang, S.; Wei, W.; Zhang, Y.; Park, H.; Roy-Chaudhuri, B.; Li, P.; Xu, J.; Chu, K.; et al. A transferRNA-derived small RNA regulates ribosome biogenesis. Nature 2017, 552, 57-62, doi:10.1038/nature25005.

36. Gill, J.; Gorlick, R. Advancing therapy for osteosarcoma. Nat. Rev. Clin. Oncol. 2021, doi:10.1038/s41571-021-00519-8.

37. Luo, J.; Yang, H.; Song, B.L. Mechanisms and regulation of cholesterol homeostasis. Nat. Rev. Mol. Cell Biol. 2020, 21, 225245, doi:10.1038/s41580-019-0190-7.

38. Lin, Y.; Huang, M.; Wang, S.; You, X.; Zhang, L.; Chen, Y. PAQR11 modulates monocyte-to-macrophage differentiation and pathogenesis of rheumatoid arthritis. Immunology 2021, 163, 60-73, doi:10.1111/imm.13303.

39. Honda, Y.; Yamagiwa, S.; Matsuda, Y.; Takamura, M.; Ichida, T.; Aoyagi, Y. Altered expression of TLR homolog RP105 on monocytes hypersensitive to LPS in patients with primary biliary cirrhosis. J. Hepatol. 2007, 47, 404-411, doi:10.1016/j.jhep.2007.03.012.

40. Xia, Z.; Wang, C.; Zhang, H. The downregulation of NCXs is positively correlated with the prognosis of stage II-IV colon cancer. World J. Surg. Oncol. 2021, 19, 177, doi:10.1186/s12957-021-02284-5.

41. Staiano, R.I.; Granata, F.; Secondo, A.; Petraroli, A.; Loffredo, S.; Annunziato, L.; Triggiani, M.; Marone, G. Human macrophages and monocytes express functional $\mathrm{Na}(+) / \mathrm{Ca}(2+)$ exchangers 1 and 3. Adv. Exp. Med. Biol. 2013, 961, 317-326, doi:10.1007/978-1-4614-4756-6_27.

42. van Hagen, P.M.; Dalm, V.A.; Staal, F.; Hofland, L.J. The role of cortistatin in the human immune system. Mol. Cell. Endocrinol. 2008, 286, 141-147, doi:10.1016/j.mce.2008.03.007.

43. Cassoni, P.; Muccioli, G.; Marrocco, T.; Volante, M.; Allia, E.; Ghigo, E.; Deghenghi, R.; Papotti, M. Cortistatin-14 inhibits cell proliferation of human thyroid carcinoma cell lines of both follicular and parafollicular origin. J. Endocrinol. Invest. 2002, 25, 362-368, doi:10.1007/bf03344019.

44. Li, M.; Yan, S.; Fisher, W.E.; Chen, C.; Yao, Q. New roles of a neuropeptide cortistatin in the immune system and cancer. World J. Surg. 2005, 29, 354-356, doi:10.1007/s00268-004-7811-8.

45. Ding, Y.L.; Sun, S.F.; Zhao, G.L. COL5A2 as a potential clinical biomarker for gastric cancer and renal metastasis. Medicine (Baltimore) 2021, 100, e24561, doi:10.1097/MD.0000000000024561.

46. Zeng, X.T.; Liu, X.P.; Liu, T.Z.; Wang, X.H. The clinical significance of COL5A2 in patients with bladder cancer: A retrospective analysis of bladder cancer gene expression data. Medicine (Baltimore) 2018, 97, e0091, doi:10.1097/MD.0000000000010091.

47. Calvo, F.; Ranftl, R.; Hooper, S.; Farrugia, A.J.; Moeendarbary, E.; Bruckbauer, A.; Batista, F.; Charras, G.; Sahai, E. Cdc42EP3/BORG2 and Septin Network Enables Mechano-transduction and the Emergence of Cancer-Associated Fibroblasts. Cell reports 2015, 13, 2699-2714, doi:10.1016/j.celrep.2015.11.052. 
48. Eduardo da Silva, L.; Russo, L.C.; Forti, F.L. Overactivated Cdc42 acts through Cdc42EP3/Borg2 and NCK to trigger DNA damage response signaling and sensitize cells to DNA-damaging agents. Exp. Cell Res. 2020, 395, 112206, doi:10.1016/j.yexcr.2020.112206.

49. Farrugia, A.J.; Calvo, F. Cdc42 regulates Cdc42EP3 function in cancer-associated fibroblasts. Small GTPases 2017, 8, 49-57, doi:10.1080/21541248.2016.1194952.

50. Chen, W.; Zhu, Y.; Zhang, W.; Zhang, H.; Zhou, Y.; Sun, P.; Wu, G. CDC42EP3 is a key promoter involved in the development and progression of gastric cancer. Carcinogenesis 2021, 42, 1179-1188, doi:10.1093/carcin/bgab048.

51. Feng, Q.; Xu, D.; Zhou, M.; Wu, Z.; Wu, Z.; Wang, Z.; Bi, J.; Pei, W. CDC42EP3 promotes colorectal cancer through regulating cell proliferation, cell apoptosis and cell migration. Cancer Cell Int. 2021, 21, doi:10.1186/s12935-021-01845-8.

52. Ezawa, I.; Sawai, Y.; Kawase, T.; Okabe, A.; Tsutsumi, S.; Ichikawa, H.; Kobayashi, Y.; Tashiro, F.; Namiki, H.; Kondo, T.; et al. Novel p53 target gene FUCA1 encodes a fucosidase and regulates growth and survival of cancer cells. Cancer Sci. 2016, 107, 734-745, doi:10.1111/cas.12933.

53. Bonin, S.; Parascandolo, A.; Aversa, C.; Barbazza, R.; Tsuchida, N.; Castellone, M.D.; Stanta, G.; Vecchio, G. Reduced expression of $\alpha$-L-Fucosidase-1 (FUCA-1) predicts recurrence and shorter cancer specific survival in luminal B LN+breast cancer patients. Oncotarget 2018, 9, 15228-15238, doi:10.18632/oncotarget.24445.

54. Vecchio, G.; Parascandolo, A.; Allocca, C.; Ugolini, C.; Basolo, F.; Moracci, M.; Strazzulli, A.; Cobucci-Ponzano, B.; Laukkanen, M.O.; Castellone, M.D.; et al. Human a-L-fucosidase-1 attenuates the invasive properties of thyroid cancer. Oncotarget 2017, 8, 27075-27092, doi:10.18632/oncotarget.15635.

55. Xu, L.; Li, Z.; Song, S.; Chen, Q.; Mo, L.; Wang, C.; Fan, W.; Yan, Y.; Tong, X.; Yan, H. Downregulation of $\alpha$-1-fucosidase 1 suppresses glioma progression by enhancing autophagy and inhibiting macrophage infiltration. Cancer Sci. 2020, 111, 22842296, doi:10.1111/cas.14427.

56. Li, H.; Han, X.; Yang, S.; Wang, Y.; Dong, Y.; Tang, T. FOXP1 drives osteosarcoma development by repressing P21 and RB transcription downstream of P53. Oncogene 2021, doi:10.1038/s41388-021-01742-4.

57. Noller, H.F. Evolution of protein synthesis from an RNA world. Cold Spring Harb. Perspect. Biol. 2012, 4, a003681, doi:10.1101/cshperspect.a003681.

58. Pelletier, J.; Thomas, G.; Volarević, S. Ribosome biogenesis in cancer: new players and therapeutic avenues. Nat. Rev. Cancer 2018, 18, 51-63, doi:10.1038/nrc.2017.104. 